\begin{tabular}{|c|c|c|c|}
\hline Figure \# & $\begin{array}{l}\text { Figure title } \\
\text { One sentence only }\end{array}$ & $\begin{array}{l}\text { Filename } \\
\text { This should be the name } \\
\text { the file is saved as when it } \\
\text { is uploaded to our system. } \\
\text { Please include the file } \\
\text { extension. i.e.: } \\
\text { Smith_ED_Fig1.jpg }\end{array}$ & $\begin{array}{l}\text { Figure Legend } \\
\text { If you are citing a reference for the first time in these legends, please include } \\
\text { all new references in the main text Methods References section, and carry on } \\
\text { the numbering from the main References section of the paper. If your paper } \\
\text { does not have a Methods section, include all new references at the end of the } \\
\text { main Reference list. }\end{array}$ \\
\hline Extended Data Fig. 1 & $\begin{array}{l}\text { Selection criteria for } \\
\text { the integrated single- } \\
\text { cell analysis and } \\
\text { gating strategies }\end{array}$ & ED_Fig1.tif & 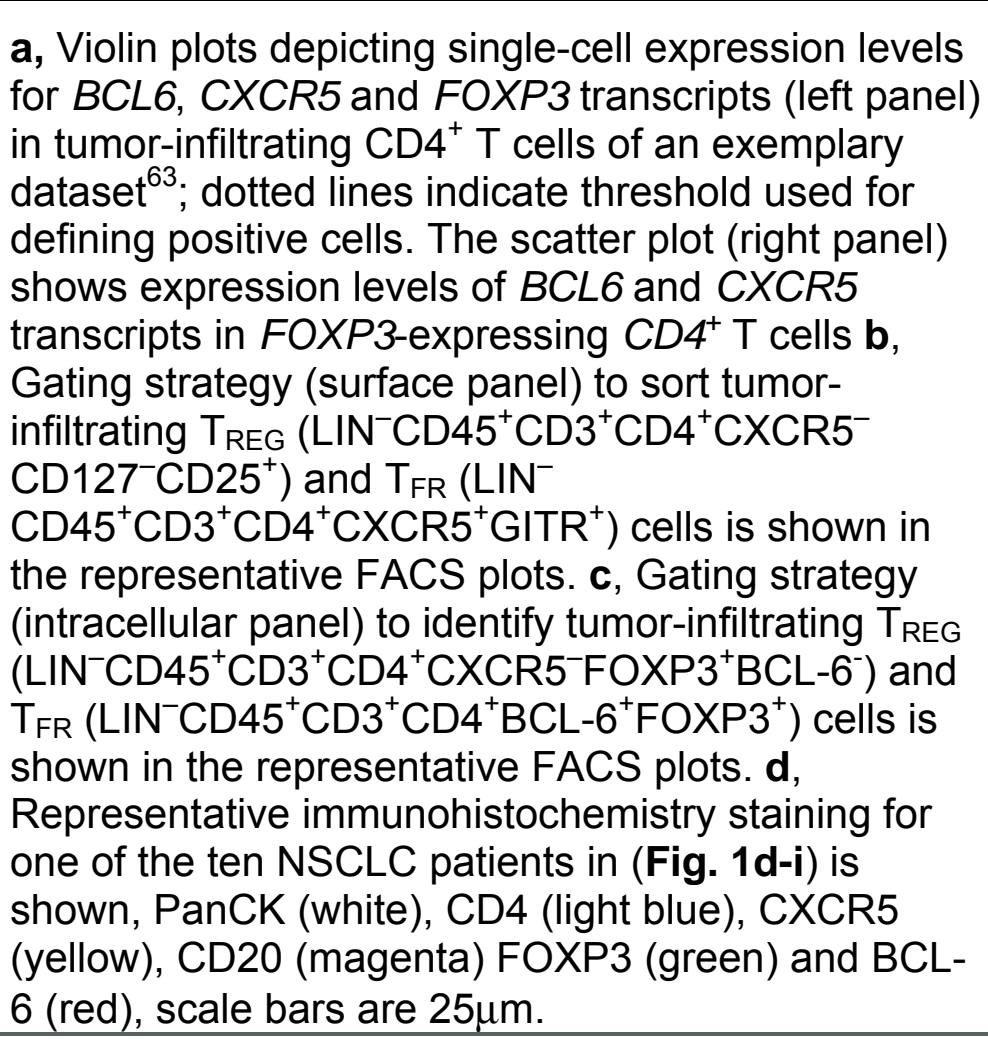 \\
\hline Extended Data Fig. 2 & $\begin{array}{l}\text { Transcriptome } \\
\text { analysis of murine } \\
\mathrm{T}_{\mathrm{FR}} \text { cells and } \\
\text { characterization of } \\
\mathrm{T}_{\mathrm{FR}} \text { cells in murine } \\
\text { tumors. }\end{array}$ & ED_Fig2.tif & 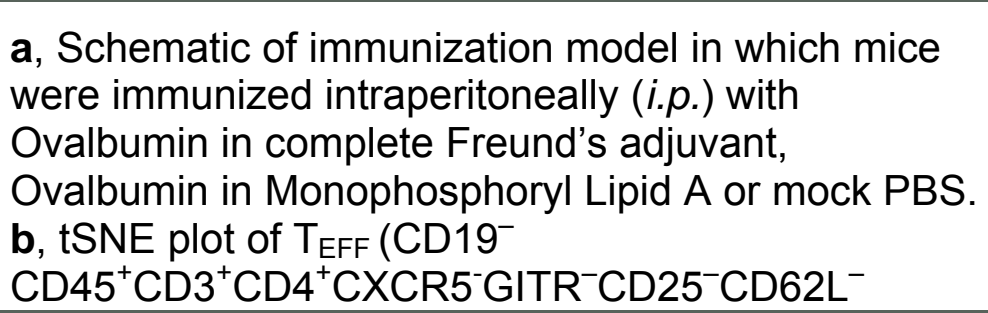 \\
\hline
\end{tabular}




\begin{tabular}{|c|c|c|c|}
\hline & & & 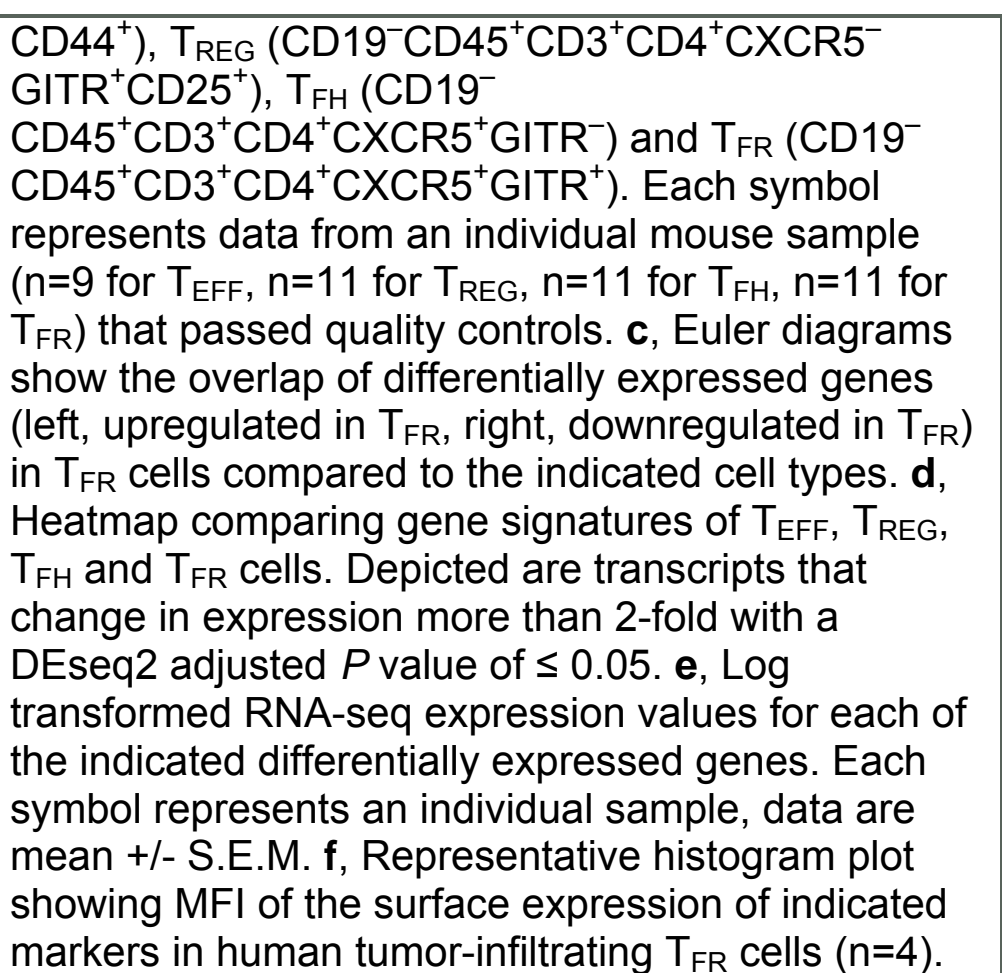 \\
\hline Extended Data Fig. 3 & $\begin{array}{l}\text { Transcriptome } \\
\text { analysis of human } \\
\text { tumor-infiltrating } \mathrm{T}_{\mathrm{FR}} \\
\text { cells. }\end{array}$ & ED_Fig3.tif & $\begin{array}{l}\text { a, Weighted gene co-expression network analysis } \\
\text { (WGCNA) depicted as a Topological Overlap Matrix } \\
\text { (TOM) heatmap. It included all genes used in the } \\
\text { WGCNA analysis and each row and column } \\
\text { correspond to a single gene. Red color indicates the } \\
\text { degree of topological overlap. The signed network } \\
\text { was generated with bulk RNA-seq data of sorted } \\
\text { cells enriched for tumor-infiltrating } \mathrm{T}_{\mathrm{REG}}\left(\mathrm{LIN}^{-}\right. \\
\left.\mathrm{CD} 45^{+} \mathrm{CD} 3^{+} \mathrm{CD} 4^{+} \mathrm{CXCR} 5^{-} \mathrm{CD} 127^{-} \mathrm{CD} 25^{+}\right) \text {and } \mathrm{T}_{\mathrm{FR}} \\
\left(\mathrm{LIN} \mathrm{IN}^{-} \mathrm{C} 45^{+} \mathrm{CD} 3^{+} \mathrm{CD} 4^{+}\right. \\
\left.\text {CXCR5 }{ }^{+} \mathrm{GITR}{ }^{+}\right) \text {populations respectively from } 10 \\
\text { treatment naïve NSCLC patients (as described in } \\
\text { Fig.2 a-d). b, Spearman correlation analysis of the }\end{array}$ \\
\hline
\end{tabular}




\begin{tabular}{|c|c|c|c|}
\hline & & & 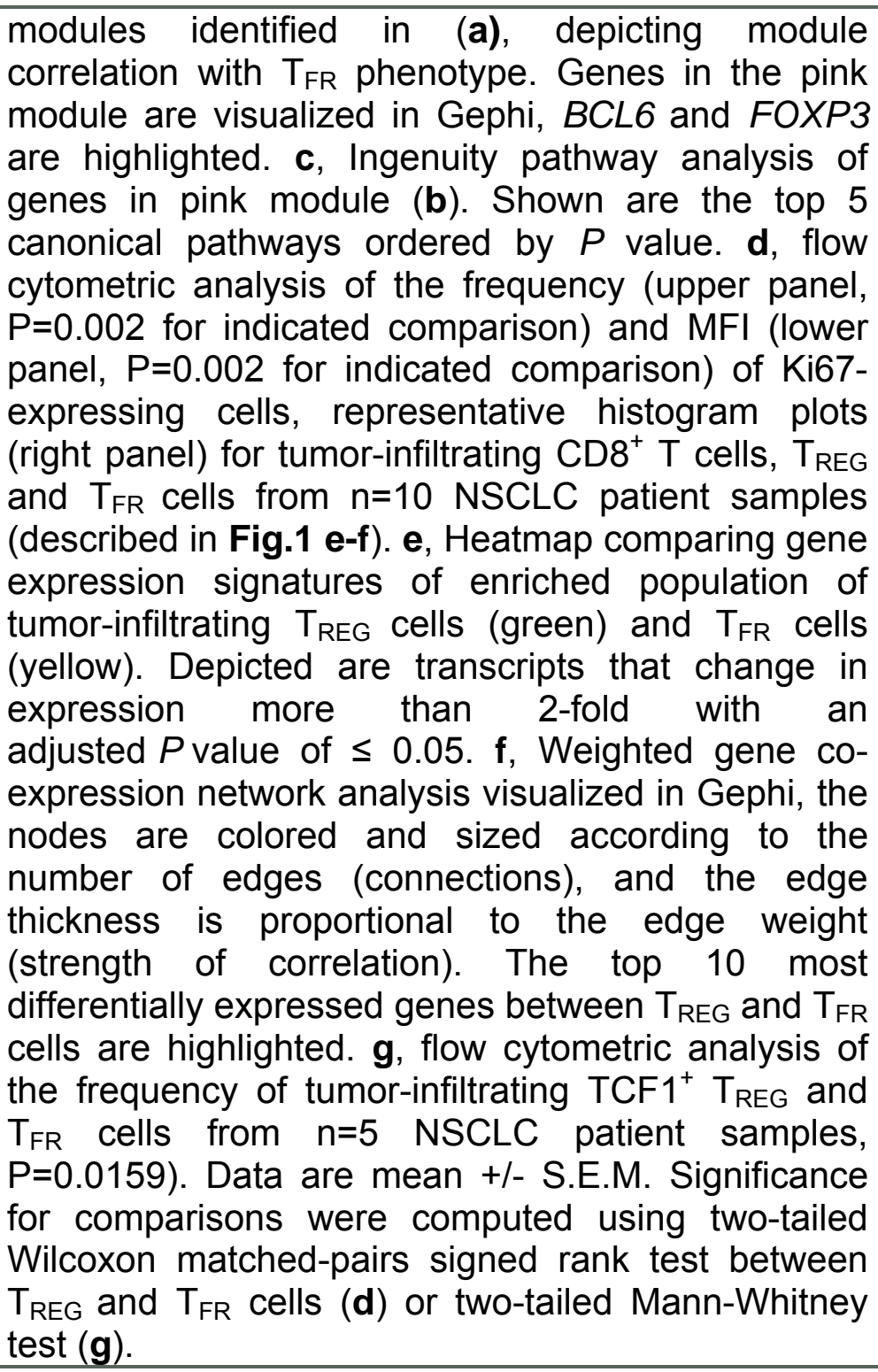 \\
\hline Extended Data Fig. 4 & Cell trajectory & ED_Fig4.tif & a, Single-cell pseudo-time trajectory of cells in \\
\hline
\end{tabular}




\begin{tabular}{|c|c|c|c|}
\hline & $\begin{array}{l}\text { analysis of human } \\
\mathrm{T}_{\mathrm{REG}} \text { and } \mathrm{T}_{\mathrm{FR}} \text { cells } \\
\text { from primary tumor } \\
\text { tissue and } \\
\text { metathesized tumor- } \\
\text { infiltrated lymph } \\
\text { nodes. }\end{array}$ & & $\begin{array}{l}\text { cluster } 1 \text { ( } T_{\mathrm{REG}} \text { cells) and cluster } 6 \text { ( } \mathrm{T}_{\mathrm{FR}} \text { cells) (left) or } \\
\text { cells from primary tumor tissue or metastatic tumor- } \\
\text { infiltrated lymph nodes (right) constructed using the } \\
\text { Monocle3 algorithm. b, Normalized gene expression } \\
\text { of IL1R2, CCR8, TNFRSF9, TNFRSF18 and PDCD1 } \\
\text { on pseudotime path as in (a). }\end{array}$ \\
\hline Extended Data Fig. 5 & $\begin{array}{l}\text { TCR-seq analysis of } \\
\text { tumor-infiltrating } \\
T_{\text {REG }} \text { and } T_{F R} \text { cells. }\end{array}$ & ED_Fig5.tif & 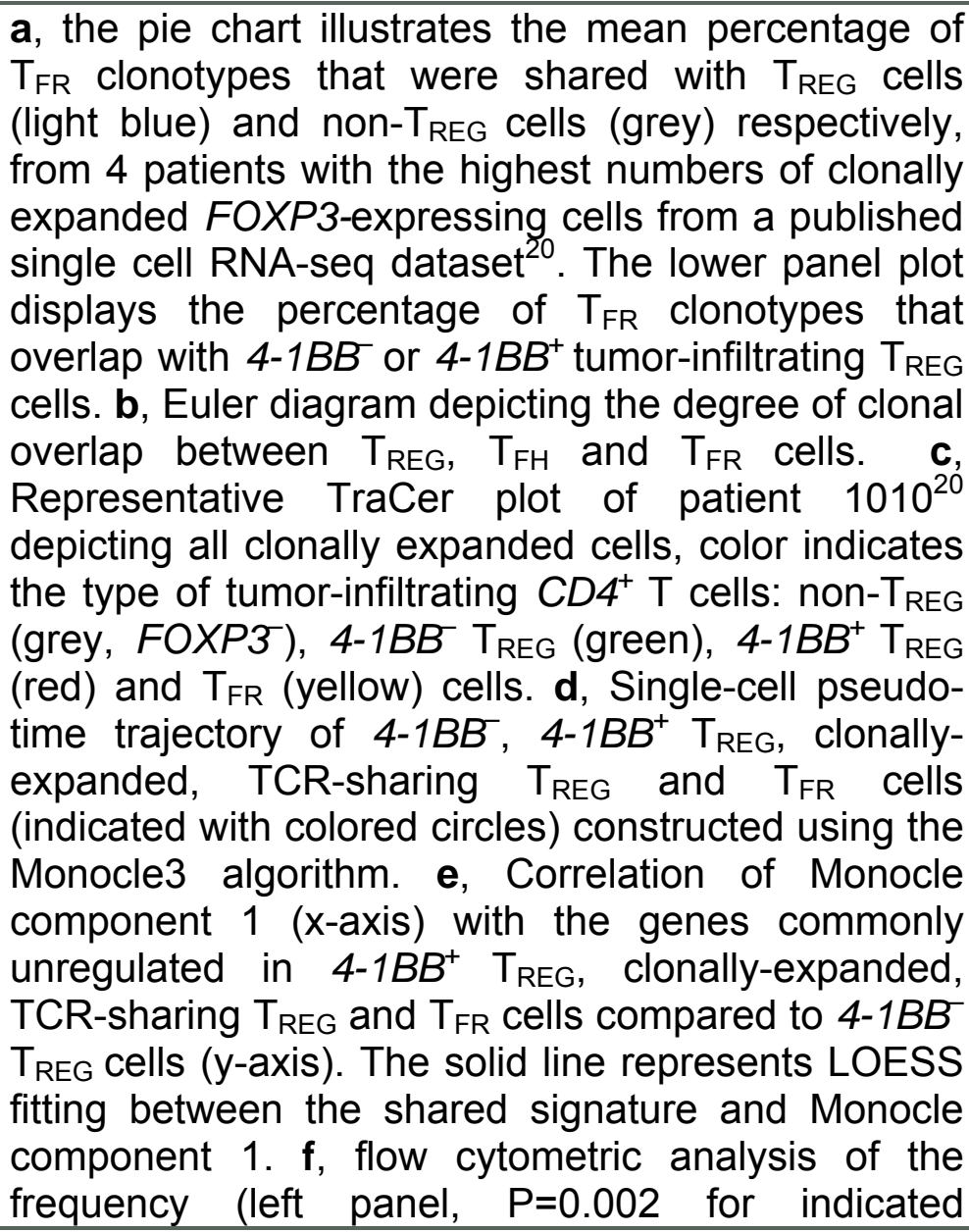 \\
\hline
\end{tabular}




\begin{tabular}{|c|c|c|c|}
\hline & & & $\begin{array}{l}\text { comparison), MFI (middle panel, } \mathrm{P}=0.002 \text { for } \\
\text { indicated comparison) for 4-1BB expression in } \\
\text { tumor-infiltrating CD8 }{ }^{+} \mathrm{T} \text { cells, } \mathrm{T}_{\mathrm{REG}} \text { and } \mathrm{T}_{\mathrm{FR}} \text { cells } \\
\text { ( } \mathrm{n}=10 \text { treatment naïve NSCLC patients as in Fig. } 2 \text { a- } \\
\mathrm{d}) \text {. Data are mean }+/- \text { S.E.M. Significance for } \\
\text { comparisons were computed using two-tailed } \\
\text { Wilcoxon matched-pairs signed rank test between } \\
\mathrm{T}_{\mathrm{REG}} \text { and } \mathrm{T}_{\mathrm{FR}} \text { cells. }\end{array}$ \\
\hline Extended Data Fig. 6 & $\begin{array}{l}\text { Characterization of } \\
\text { murine } T_{F R} \text { cells in } \\
\text { immunization and } \\
\text { cancer setting. }\end{array}$ & ED_Fig6.tif & 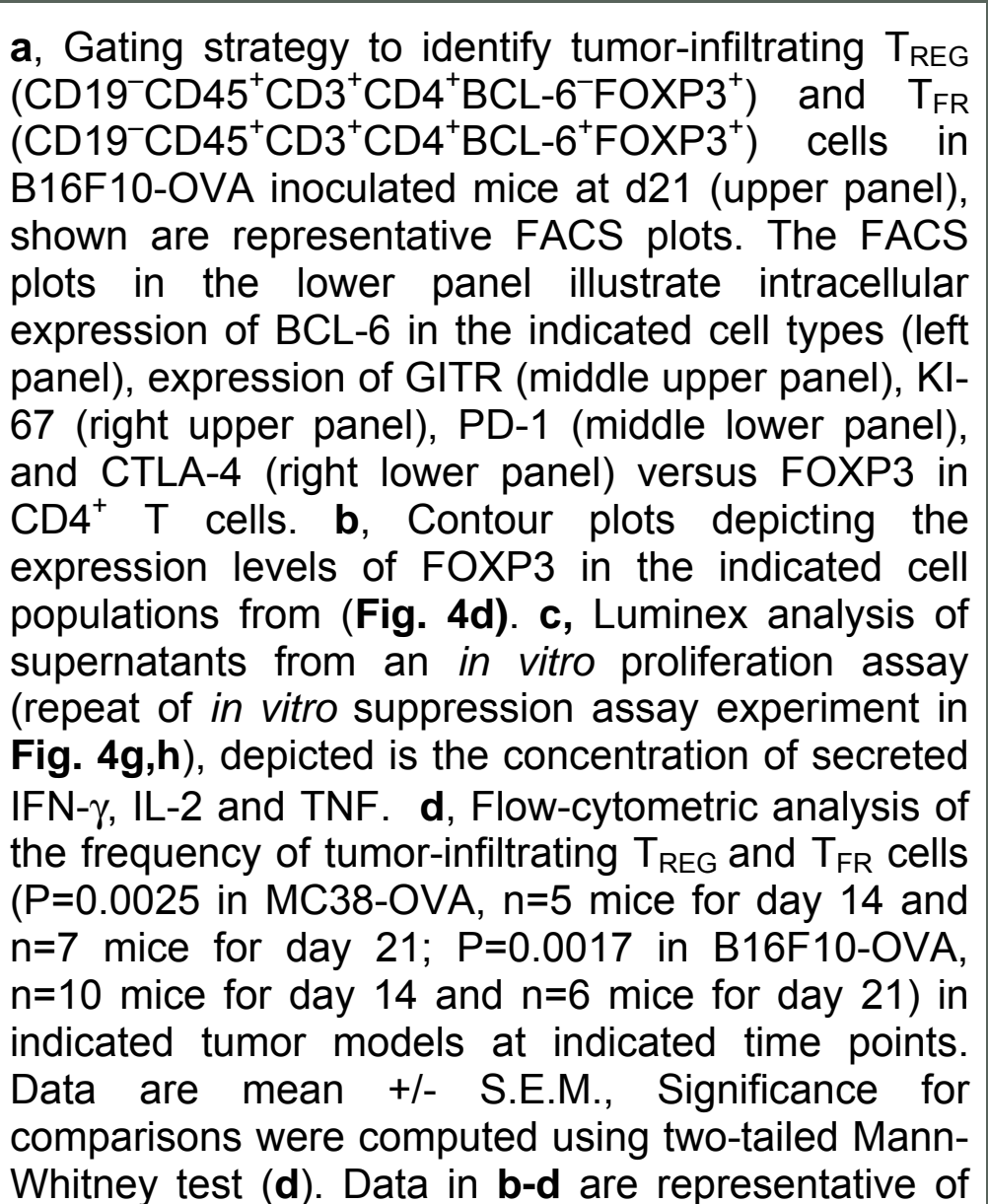 \\
\hline
\end{tabular}




\begin{tabular}{|c|c|c|c|}
\hline & & & two independent experiments. \\
\hline Extended Data Fig. 7 & $\begin{array}{l}\text { Human } T_{F R} \text { cells are } \\
\text { responsive to anti- } \\
\text { PD-1 therapy. }\end{array}$ & ED_Fig7.tif & 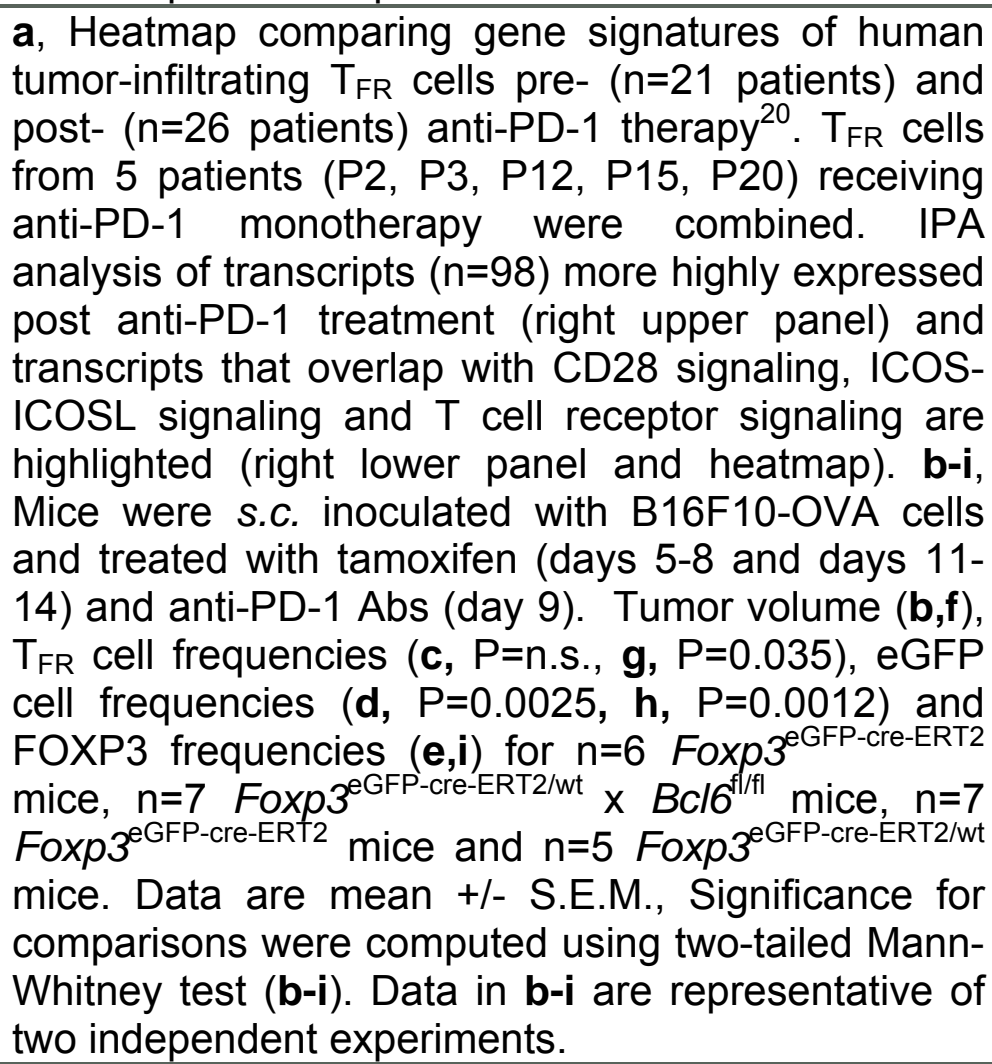 \\
\hline Extended Data Fig. 8 & $\begin{array}{l}\text { Murine } T_{F R} \text { cells are } \\
\text { depleted by anti- } \\
\text { CTLA-4 thereapy }\end{array}$ & ED_Fig8.tif & 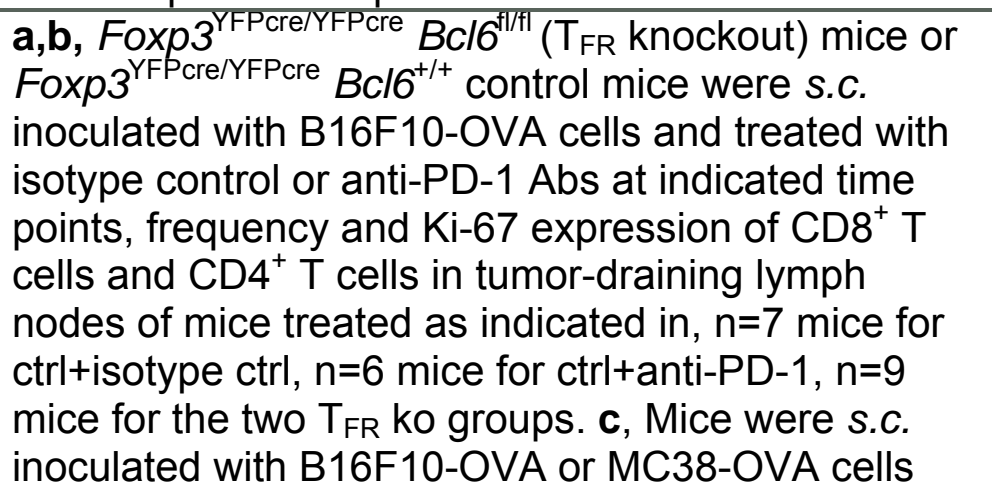 \\
\hline
\end{tabular}




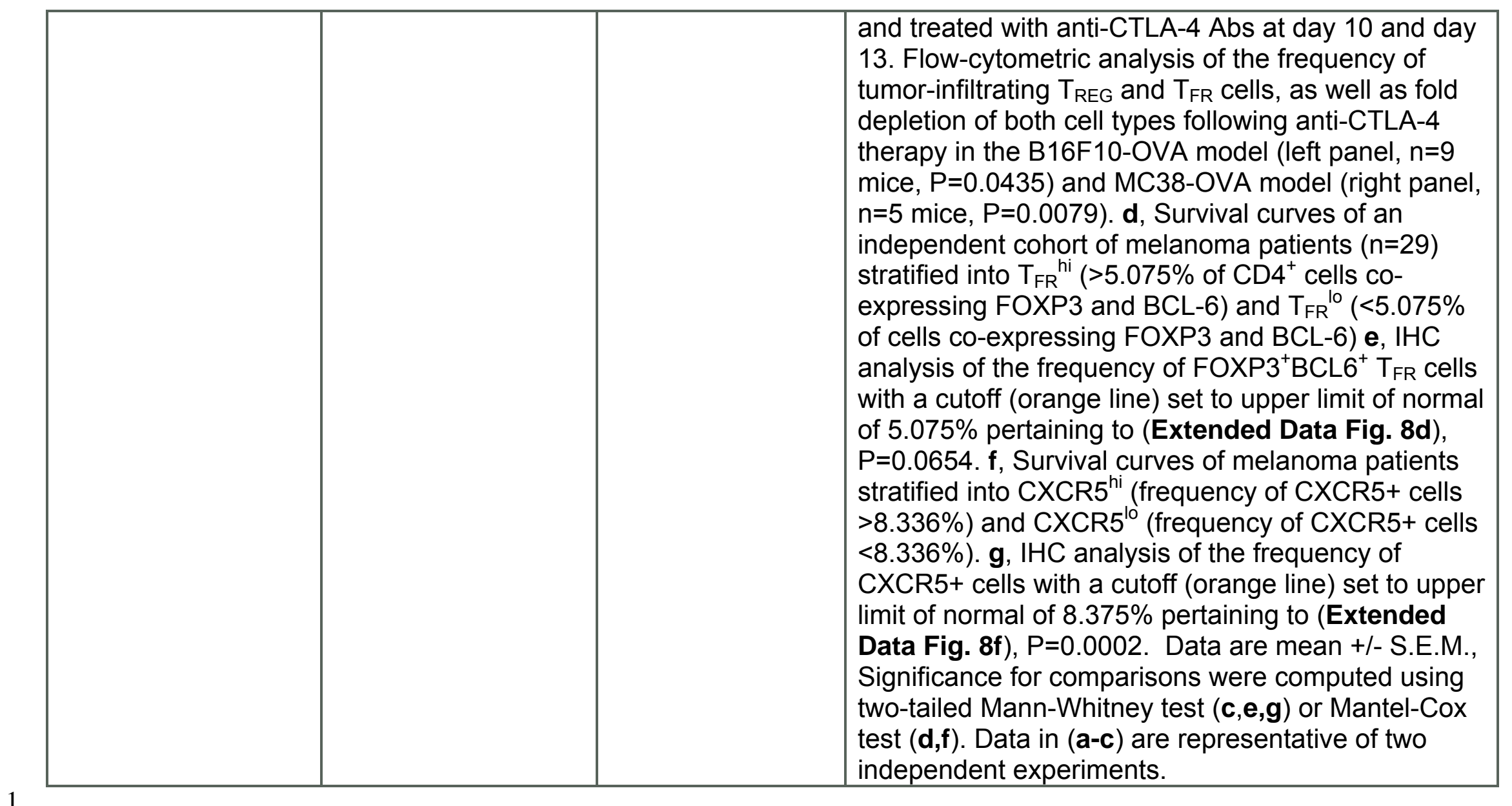

1

\begin{tabular}{|l|l|l|l|}
\hline Item & Present? & $\begin{array}{l}\text { Filename } \\
\text { This should be the name the file } \\
\text { is saved as when it is uploaded } \\
\text { to our system, and should } \\
\text { include the file extension. The } \\
\text { extension must be .pdf }\end{array}$ & $\begin{array}{l}\text { A brief, numerical description of file contents. } \\
\text { i.e.: Supplementary Figures 1-4, Supplementary Discussion, and } \\
\text { Supplementary Tables 1-4. }\end{array}$ \\
\hline Supplementary Information & No & & \\
\hline
\end{tabular}




\begin{tabular}{|l|l|l|}
\hline Reporting Summary & Yes & Reporting summary.pdf \\
\hline Peer Review Information & Yes & OFFICE USE ONLY \\
\hline
\end{tabular}

\begin{tabular}{|l|l|l|}
\hline Parent Figure or Table & $\begin{array}{l}\text { Filename } \\
\text { This should be the name the file is saved as when it } \\
\text { is uploaded to our system, and should include the } \\
\text { file extension. i.e.: Smith_SourceData_Fig1.xls, or } \\
\text { Smith_} \\
\text { Unmodified_Gels_Fig1.pdf }\end{array}$ & $\begin{array}{l}\text { Data description } \\
\text { i.e.: Unprocessed Western Blots and/or gels, Statistical Source Data, etc. }\end{array}$ \\
\hline Source Data Fig. 1 & SourceData_Fig1.xlsx & Source Data Integrated analysis \\
\hline Source Data Fig. 2 & SourceData_Fig2.xlsx & Statistical Source Data \\
\hline Source Data Fig. 3 & SourceData_Fig3.xlsx & Statistical Source Data \\
\hline Source Data Fig. 4 & SourceData_Fig4.xlsx & Statistical Source Data \\
\hline Source Data Fig. 5 & SourceData_Fig5.xlsx & Statistical Source Data \\
\hline Source Data Fig. 6 & SourceData_Fig6.xlsx & Statistical Source Data \\
\hline Source Data Fig. 7 & SourceData_Fig7.xlsx & Statistical Source Data \\
\hline Source Data Extended Data Fig. 2 & SourceData_ED_Fig2.xlsx & Statistical Source Data \\
\hline Source Data Extended Data Fig. 3 & SourceData_ED_Fig3.xlsx & Statistical Source Data \\
\hline Source Data Extended Data Fig. 5 & SourceData_ED_Fig5.xlsx & Statistical Source Data \\
\hline Source Data Extended Data Fig. 6 & SourceData_ED_Fig6.xlsx & Statistical Source Data \\
\hline Source Data Extended Data Fig. 7 & SourceData_ED_Fig7.xlsx & Statistical Source Data \\
\hline Source Data Extended Data Fig. 8 & SourceData_ED_Fig8.xlsx & Statistical Source Data \\
\hline
\end{tabular}




\section{Intratumoral follicular regulatory T cells curtail anti-PD-1 treatment efficacy}

11

12 Simon Eschweiler ${ }^{1}$, James Clarke ${ }^{1}$, Ciro Ramírez-Suástegui ${ }^{1}$, Bharat Panwar ${ }^{1}$, Ariel Madrigal ${ }^{1}$, Serena J Chee ${ }^{2,3,4}$, Ioannis Karydis $^{2,4}$,

13 Edwin Woo $^{4}$, Aiman Alzetani ${ }^{4}$, Somaia Elsheikh ${ }^{5,6}$, C.J. Hanley ${ }^{2,3}$, G.J. Thomas ${ }^{2,3}$, Peter S Friedmann ${ }^{7}$, Tilman Sanchez-Elsner ${ }^{3,7}$,

14 Ferhat $\mathrm{Ay}^{1,8}$, Christian H Ottensmeier ${ }^{1,2,3,4,10}$, Pandurangan Vijayanand ${ }^{1,3,7,9,10, *}$

15

$16 \quad{ }^{1}$ La Jolla Institute for Immunology, La Jolla, CA, USA.

$17{ }^{2}$ NIHR and CRUK Southampton Experimental Cancer Medicine Center, Faculty of Medicine, University of Southampton,

18 Southampton, UK.

$19{ }^{3}$ NIHR Southampton Biomedical Research Center, Faculty of Medicine, University of Southampton, Southampton, UK.

$20{ }^{4}$ Southampton University Hospitals NHS Foundation Trust, Southampton, UK.

$21{ }^{5}$ Department of Cellular Pathology, Nottingham University Hospital

$22{ }^{6}$ Division of Cancer and Stem cells, School of Medicine, University of Nottingham

$23{ }^{7}$ Clinical and Experimental Sciences, University of Southampton, Faculty of Medicine, Southampton UK.

$24{ }^{8}$ Department of Pediatrics, University of California San Diego, La Jolla, CA, USA

$25{ }^{9}$ Department of Medicine, University of California San Diego, La Jolla, CA, USA.

$26 \quad{ }^{10}$ These authors jointly directed the work 
8

*Correspondence: vijay@lji.org

\section{Abstract}

Immune checkpoint blockade (ICB) has shown remarkable clinical success in boosting anti-tumor immunity. However, the breadth of its cellular targets and specific mode of action remain elusive. We find that tumor-infiltrating $T$ follicular regulatory ( $\left.T_{F R}\right)$ cells were prevalent in tumor tissues of several cancer types. They were primarily located within tertiary lymphoid structures and exhibited superior suppressive capacity and in vivo persistence when compared to $\mathrm{T}_{\mathrm{REG}}$ cells, with whom they shared a clonal and developmental relationship. In syngeneic tumor models, anti-PD-1 treatment increased the number of tumor-infiltrating $T_{F R}$ cells. $T_{F R}$ cell deficiency or depletion of $\mathrm{T}_{\mathrm{FR}}$ cells with anti-CTLA-4 antibody prior to anti-PD-1 treatment, improved tumor control in mice.

Notably, in a cohort of 271 melanoma patients, treatment with anti-CTLA-4 followed by anti-PD-1 at progression was associated with better survival outcomes than monotherapy with anti-PD-1 or anti-CTLA-4, anti-PD-1 followed by anti-CTLA-4 at progression or concomitant combination therapy. 


\section{Introduction}

61 An increased density of $T$ regulatory $\left(T_{R E G}\right)$ cells in tumors has been linked to poor survival outcomes ${ }^{1}$. In secondary lymphoid

62 organs, $T_{R E G}$ cells have been shown to differentiate into PD-1 expressing $T$ follicular regulatory cells ( $\left.T_{F R}\right)$ that restrain germinal

63 center responses ${ }^{2}$, impede humoral immunity towards self-antigens and display heightened suppressive capacity when compared to

$64 \mathrm{~T}_{\mathrm{REG}}$ cells $\mathrm{s}^{3,4}$. $\mathrm{T}_{\mathrm{FR}}$ cells are being characterized by their joint expression of the surface molecules CXCR5 and GITR ${ }^{2,5}$, or by their co-

65 expression of the transcription factors FOXP3 and BCL- $6^{6}$. Several studies have demonstrated that, depending on disease context 66 and organ, cells of the T follicular lineage express varying levels of CXCR5 and BCL- $6^{7,8}$. Notably, it has been shown that deletion of

67 CXCR5 expression in FOXP3-expressing cells does not abrogate the development and maintenance of $B C L-6^{+} T_{F R}$ cells ${ }^{6}$, indicating 
$69 \mathrm{CD} 25^{9,10}$.

While the role of $T$ follicular helper $\left(\mathrm{T}_{\mathrm{FH}}\right)$ cells, B cells, and tertiary lymphoid structures (TLS) in driving anti-tumor immune responses and responsiveness to anti-PD1 therapy is now beginning to be elucidated ${ }^{11-14}$, few studies have examined potential effects of anti-PD-1 therapy on the regulatory $\mathrm{T}$ cell compartment. Accordingly, $\mathrm{T}_{\mathrm{FR}}$ cells, their functional role in cancer, and their responsiveness to ICB have been completely overlooked so far. Based on the well-described functions of $T_{F R}$ cells in secondary lymphoid organs, we hypothesized that $\mathrm{T}_{\mathrm{FR}}$ cells are likely to be present in the TLS of tumors and modulate immune responses in the tumor microenvironment (TME). Moreover, as $\mathrm{T}_{\mathrm{FR}}$ cells have a skewed TCR repertoire towards self-antigens and because cancerous cells frequently express self or altered-self antigens, we hypothesized that $T_{R E G}$ and $T_{F R}$ cells accumulate in parallel in the $T M E$ as a means of effective immune evasion.

Herein, we report that $\mathrm{T}_{\mathrm{FR}}$ cells account for a substantial proportion of tumor-infiltrating $\mathrm{CD}^{+} \mathrm{T}$ cells, and importantly, that they are highly responsive to ICB. We further demonstrate that $T_{F R}$ cells are highly suppressive, are prevalent in multiple different cancer types, and accumulate in tumor tissues over time, likely mediated by their higher proliferative capacity and persistence in vivo that is dependent on BCL-6. Depleting $\mathrm{T}_{\mathrm{FR}}$ cells or blocking their activity with anti-CTLA-4 antibodies prior to anti-PD-1 therapy, improved efficacy of anti-PD-1 treatment in mouse tumor models and was also associated with better survival outcomes in a large cohort of melanoma patients. Finally, we found that $T_{F R}$ cells, but not $T_{R E G}$ cells, were enriched within TLS, suggesting that $T_{F R}$ cells might also impair patient survival and impede immunotherapy treatment efficacy by regulating TLS, consistent with their welldescribed role in secondary lymphoid organs ${ }^{3,4}$. Our findings thus challenge the current clinical practice of unselective administration 
of anti-PD-1 therapy, which hence overlooks any possibility for its potential to impede anti-tumor immune responses. By elucidating

87 the functional properties of intratumoral $T_{F R}$ cells and by identifying them as one of the major targets of ICB, we provide critical insights into how anti-CTLA-4 and anti-PD-1 therapies mediate their function and highlight the potential clinical benefit of depleting intratumoral $\mathrm{T}_{\mathrm{FR}}$ cells prior to initiation of anti-PD1 therapy.

90

91

\section{Results}

92

\section{$T_{F R}$ cells are present in multiple cancer types}

We integrated 9 published single-cell RNA-seq datasets and performed a meta-analysis of tumor-infiltrating CD4 ${ }^{+} \mathrm{T}_{\text {cells }}$ $(n=25,149)$ from patients with six different cancer types (Table 1). As expected, FOXP3-expressing CD4 ${ }^{+} \mathrm{T}_{\text {cells }}$ (i.e., $\mathrm{T}_{\mathrm{REG}}$ cells) clustered distinctly and represented $5-55 \%$ of all tumor-infiltrating CD4 ${ }^{+}$T cells (Fig. 1a,b). We found that a substantial proportion (5$30 \%$ in all tumor types) of FOXP3-expressing CD4 ${ }^{+}$T cells co-expressed BCL6 and/or CXCR5 (Fig. 1c and Extended Data Fig. 1a), which encode for markers indicative of cells of a follicular lineage in humans and mice ${ }^{2,15}$, and thus represent tumor-infiltrating $T_{F R}$ cells, an important regulatory subset that has not been appreciated so far. We confirmed the presence $(\sim 10-20 \%$ of all tumorinfiltrating $\mathrm{CD}^{+} \mathrm{T}$ cells) and localization of $\mathrm{T}_{\mathrm{FR}}$ cells in tumor samples from patients with treatment-naïve early-stage non-small cell lung cancer (NSCLC) by multi-parameter flow cytometry and immunohistochemistry (Fig.2a-f, Extended Data Fig. 1b-d and Table 2). $T_{F R}$ cells, like $T_{R E G}$ cells, maintained surface expression of CD25 and ICOS (Fig. 2b). To determine if currently available immunotherapies, like anti-CTLA4 and anti-PD1 therapies, also target tumor-infiltrating $\mathrm{T}_{\mathrm{FR}}$ cells, we assessed their expression of CTLA-4 and PD-1. Notably, $T_{F R}$ cells expressed the highest levels of CTLA-4 and PD-1 among all tumor infiltrating T cells (Fig. 2c,d), suggesting that anti-CTLA-4 can more efficiently target $T_{F R}$ cells, and that anti-PD-1 therapies may inadvertently activate such 
suppressive $\mathrm{T}_{\mathrm{FR}}$ cells. A fraction $(\sim 15 \%)$ of all tumor-infiltrating $C D 4^{+} \mathrm{T}$ cells exhibited a $\mathrm{T}_{\mathrm{FH}}$ cell phenotype (Fig. 2a) that lacked expression of CD25, but expressed ICOS, CTLA-4 and PD-1 (Fig. 2b-d). Given the recent findings highlighting the importance of B cells, $\mathrm{T}_{\mathrm{FH}}$ cells and TLS with regard to heightened anti-tumor immunity, improved patient survival and responsiveness to immunotherapy ${ }^{11-14}$, we next assessed the cellular context in which $T_{F R}$ cells exert their function within the TME. Multicolorimmunohistochemistry analyses confirmed the presence of $T_{F R}$ cells in tumor tissues (Fig. 2e and Extended Data Fig. 1d). Crucially, we found that $T_{F R}$ cells, unlike $T_{R E G}$ cells, were predominantly located within TLS (Fig. 2f), indicating that $T_{F R}$ cells might inhibit antitumor immunity by impeding the function of cells in their vicinity (i.e., of ectopic $\mathrm{B}$ and $\mathrm{T}_{\mathrm{FH}}$ cell responses) or by regulating TLS formation or maintenance.

\section{$T_{F R}$ cells exhibit unique transcriptomic features}

As few studies have thoroughly analyzed the transcriptomic features of $T_{F R}$ cells, we first utilized well-established immunization models in mice to gain mechanistic insights into $T_{F R}$ cell function and to assess whether the features identified in human tumor-infiltrating $\mathrm{T}_{\mathrm{FR}}$ cells are also applicable to murine $\mathrm{T}_{\mathrm{FR}}$ cells. Immunization with ovalbumin and adjuvant (CFA or MPLA) induced robust $T_{F R}$ responses (Extended Data Fig. 2a). Comparative analysis of their transcriptome with that of other $T_{H}$ subsets showed increased expression of many transcripts specifically in $T_{F R}$ cells $(n=84)$, (Extended Data Fig. 2b-e and Table 3), and notably the transcripts enriched in $T_{R E G}$ cells compared to both $T_{F H}$ and $T_{E F F}$ populations $(n=127)$ were also highly expressed in $T_{F R}$ cells (Extended Data Fig. 2d,e). These include several transcripts (e.g., Tnfrsf1 $b^{16}$, Lag $3^{17}$, Tigit $^{18}$, Batf ${ }^{19}$, and $/ / 1$ r2 ${ }^{1,20}$ ) encoding for products associated with heightened suppressive capacity; Ccr8, which was associated with particularly poor clinical outcomes in cancer $^{1,21}$, and genes associated with $\mathrm{CD}^{+} \mathrm{T}$ cell dysfunction and survival ${ }^{22}$ (Pdcd1 and Tox) (Extended Data Fig. 2d,e). The 
protein expression levels of some of these molecules, e.g., TNFR2 (encoded by TNFRSF1B), LAG3, TIGIT and CCR8, were confirmed in human tumor-infiltrating $T_{F R}$ cells (Extended Data Fig. 2f), suggesting suppressive capacity of $T_{F R}$ cells and likely conservation of functional potential across species.

We next performed bulk RNA-seq analyses of enriched populations of $\mathrm{T}_{\mathrm{REG}}\left(\mathrm{CD} 4^{+} \mathrm{CD} 25^{+} \mathrm{CXCR} 5^{-}\right)$and $\mathrm{T}_{\mathrm{FR}}$ cells $\left(\mathrm{CD}^{+} \mathrm{CXCR} 5^{+} \mathrm{GITR}^{+}\right)$(Extended Data Fig. 1b) isolated from tumor samples of NSCLC patients (Table 4). Weighted gene coexpression network analysis (WGCNA) (Extended Data Fig. 3a and Table 5) of bulk-sorted human $\mathrm{T}_{\mathrm{REG}}\left(\mathrm{CD} 4^{+} \mathrm{CD} 25^{+} \mathrm{CXCR} 5^{-}\right)$and $\mathrm{T}_{\mathrm{FR}}$ cells $\left(\mathrm{CD} 4^{+} \mathrm{CXCR}{ }^{+} \mathrm{GITR}^{+}\right)$(Extended Data Fig. 1b) identified a module (pink) that was positively correlated with the $\mathrm{T}_{\mathrm{FR}}$ phenotype (Extended Data Fig. 3b). Importantly, this module contained both BCL6 and FOXP3, demonstrating the linked expression of these genes, specifically in $\mathrm{T}_{\mathrm{FR}}$ cells. Ingenuity Pathway Analysis (IPA) of the pink module (Table 6) (module positively correlated with $\mathrm{T}_{\mathrm{FR}}$ phenotype) identified substantial enrichment of genes involved in cell cycle, transcriptional and translational activity and mTOR signaling, indicative of increased $T_{F R}$ cell proliferation and activity (Extended Data Fig. 3c). We confirmed that $T_{F R}$ cells indeed showed greater cell proliferation in the TME as evidenced by increased KI-67 staining (Extended Data Fig. 3d). Differential gene expression analysis of enriched populations of $T_{F R}$ cells and $T_{R E G}$ cells identified over 100 transcripts that were expressed at higher levels in $\mathrm{T}_{\mathrm{FR}}$ cells (Extended Data Fig. 3e and Table 4). Co-expression analysis of these differentially expressed transcripts revealed a number of highly correlated novel genes (e.g. DUSP14, CLP1), which may play a role in $\mathrm{T}_{\mathrm{FR}}$ cell function. Moreover, we identified TCF7 (encoding TCF-1) as a highly connected hub gene in this transcriptomic network (Extended Data Fig. 3f) and confirmed that the proportion of TCF-1-expressing cells was higher in $T_{F R}$ cells compared to $T_{R E G}$ cells (Extended Data Fig. 3g). Interestingly, TCF-1-expressing CD8 ${ }^{+}$CTLs have recently been recognized for their ability for self-renewal, stem-like 
142 properties ${ }^{23,24}$, and their pivotal role in mediating anti-cancer immune attack induced by anti-PD-1 immunotherapy ${ }^{25,26}$, suggesting

143 that TCF-1 expression might confer similar features on $T_{F R}$ cells. Together, these data indicate that intratumoral $T_{R E G}$ and $T_{F R}$ cells

144 differ in their molecular profile and demonstrate that $T_{F R}$ cells are highly proliferative in tumor tissue.

145

\section{Intratumoral $\mathrm{T}_{\mathrm{REG}}$ and $\mathrm{T}_{\mathrm{FR}}$ cells are clonally and developmentally related}

Recent data demonstrate that tumor-infiltrating $\mathrm{T}_{\mathrm{REG}}$ cells potently recognize tumor (neo)antigens and, upon antigen-

148 encounter, undergo clonal expansion ${ }^{27}$. Given that antigen-specific activation of $T_{R E G}$ cells in the context of viral infection has been

149 implicated in promoting their differentiation into $T_{F R}$ cells via TCF-1-mediated induction of BCL-6 $6^{28}$, we hypothesized that tumor-

150 associated antigen (TAA) recognition may also trigger $T_{R E G}$ to $T_{F R}$ conversion within the TME. To assess this, we performed

151 combined single-cell RNA-seq and TCR-seq of sorted $C D 4^{+}\left(T_{F H}, T_{R E G}\right.$ and $\left.T_{F R}\right)$ and CD8 ${ }^{+}$TILs from primary tumor tissue and tumor-

152 infiltrated lymph nodes of two HNSCC patients $\left(\mathrm{n}=8,722\right.$ cells). Unsupervised clustering revealed two distinct CD4 ${ }^{+} \mathrm{T}$ cell clusters (1

153 and 6) that were enriched for FOXP3 expression (Fig. 3a), and which exhibited distinct transcriptomic signatures (Fig. 3b and Table

154 7). Gene set enrichment analysis showed that cells in cluster 6 (yellow) were significantly enriched for follicular (Fig. 3c) and $T_{\mathrm{FR}}$ cell

155 signatures (Fig. 3d), thus characterizing $T_{F R}$ cells, while cells in cluster 1 (green) depict $T_{R E G}$ cells. Pathway analysis of the

156 differentially expressed genes (Fig. 3b) between $T_{F R}$ and $T_{R E G}$ cells showed enrichment for transcripts linked to metabolism, cell

157 activation and co-stimulation (Fig. 3e). Moreover, $T_{F R}$ cells expressed higher levels of transcripts liked to $T_{F R}$ function and

158 suppressive capacity (e.g., CTLA4, IL10, TGFB1, TNFRSF9, or IL1R2), and cell cycle genes (TOP2A, MKI67) (Fig. 3f). Accordingly,

159 although $T_{R E G}$ and $T_{F R}$ cells shared clonotypes (Fig. 3g,h and Table 8), $T_{F R}$ cells were more clonally expanded than $T_{R E G}$ cells (Fig.

160 3i). Importantly, TCR sharing and trajectory analysis of cells in the FOXP3-enriched clusters indicate intratumoral conversion of $T_{R E G}$ 
161 to $\mathrm{T}_{\mathrm{FR}}$ cells (Fig. 3g,h and Extended Data Fig. 4a,b). To further substantiate this notion, we re-analyzed one of the largest single-cell

162 RNA-seq datasets ${ }^{20}$ of tumor-infiltrating $\mathrm{CD}^{+} \mathrm{T}$ cells (Table 9), showing that the majority of clonally-expanded $\mathrm{T}_{\mathrm{FR}}$ clonotypes

$163(\sim 93 \%)$ were shared with $T_{\text {REG }}$ cells (Extended Data Fig. 5a, upper panel), but not $T_{F H}$ cells (Extended Data Fig. 5b). Furthermore,

$164 \mathrm{~T}_{\mathrm{REG}}$ cells that shared clonotypes with $\mathrm{T}_{\mathrm{FR}}$ cells predominantly expressed 4-1BB (TNFRSF9) transcripts (Extended Data Fig. 5a,

165 lower panel and Extended Data Fig. 5c), implying recent TCR activation ${ }^{29}$, and indicative of potential intratumoral conversion of

166 TAA-activated $T_{\text {REG }}$ to $T_{F R}$ cells. Trajectory analysis implies that 4-1BB ${ }^{+} T_{\text {REG }}$ cells (TAA-experienced) and $T_{R E G}$ cells sharing TCRs

167 with clonally-expanded $T_{F R}$ cells (purple) depict transitional states during differentiation of $T_{R E G}$ cells into $T_{F R}$ cells (Extended Data

168 Fig. 5d). Importantly, transcripts linked to cell activation, co-stimulation and suppressive function (Fig. 3j and Table 10) were

169 expressed at higher levels in $4-1 B B^{+} T_{R E G}$ cells (red), TCR sharing $T_{R E G}$ cells (purple) and clonally-expanded $T_{F R}$ cells (yellow)

170 compared to $4-1 B B^{-} \mathrm{T}_{\mathrm{REG}}$ cells (green), a gene signature that was highly associated with Monocle component 1 (Fig. $3 \mathbf{j}$ and

171 Extended Data Fig. 5e). When compared to 4-1BB ${ }^{-} T_{R E G}$ cells, $T_{F R}$ cells and $T_{R E G}$ cells on their trajectory to differentiate into $T_{F R}$ cells

172 also showed significant downregulation of CCR7 and S1PR1, genes that encode receptors required for tissue egress, suggesting

173 tissue residency of $\mathrm{T}_{\mathrm{FR}}$ cells ${ }^{30}$ (Fig. 3j).

174 These observations are consistent with a model in which the TME is initially infiltrated by a large and highly diverse pool of

175 bystander (i.e., not TAA-specific) $T_{R E G}$ cells, and a smaller pool of TAA-specific $T_{R E G}$ clones, which are poised for differentiation into

176 tissue resident $T_{F R}$ cells. This implies that $T_{F R}$ cells comprise a larger proportion of tumor-reactive clones than $T_{R E G}$ cells, a notion

177 substantiated by our finding that $T_{F R}$ cells expressed significantly higher levels of 4-1BB than $T_{R E G}$ cells (Extended Data Fig. 5f) and

178 also by the higher degree of clonal expansion (Fig. 3i).

179 


\section{$T_{F R}$ cells exhibit superior suppressive capacity}

Next, we assessed frequency, activity and functional responsiveness of $T_{F R}$ cells in murine tumor models. $T_{F R}$ cells $\left(\mathrm{CD}^{+}{ }^{+} \mathrm{CD} 4^{+} \mathrm{BCL}-6^{+} \mathrm{FOXP}^{+}\right)$were present in tumor samples from two syngeneic tumor model systems (B16F10 melanoma and MC38 colorectal tumor cell lines) (Fig. 4a and Extended Data Fig. 6a), but importantly lacked expression of CXCR5. Notably, recent studies demonstrated that ablation of CXCR5 expression in FOXP3 ${ }^{+} \mathrm{T}$ cells did not abrogate the development of BCL- $6^{+} \mathrm{T}_{\mathrm{FR}}$ cells, which still entered the germinal center reaction ${ }^{6}$. Thus, BCL- 6 expression in $\mathrm{FOXP3}^{+}$cells delineates $\mathrm{T}_{\mathrm{FR}}$ cells even in the absence of CXCR5. Similar to human $T_{F R}$ cells, murine $T_{F R}$ cells exhibited increased proliferative potential, as evidenced by KI-67 expression levels, and increased expression of TCF-1 and 4-1BB compared to $\mathrm{T}_{\mathrm{REG}}$ cells (Fig. $\mathbf{4 b}$ ). Interestingly, $\mathrm{T}_{\mathrm{FR}}$ cells also expressed significantly higher levels of the transcription factor TOX (Fig. 4c). Given that TOX was recently shown to be essential for the function and survival of TCF-1-expressing $\mathrm{CD}^{+} \mathrm{T}$ cells following chronic antigen-exposure, we speculate that TOX expression in $\mathrm{T}_{\mathrm{FR}}$ cells may help maintain their superior functionality in the face of sustained stimulation by TAA ${ }^{31,32}$.

To experimentally validate that $T_{F R}$ cells are more suppressive than $T_{R E G}$ cells, we performed functional assays in vitro and in vivo. Strikingly, we found that $T_{F R}$ cells inhibited $C D 8^{+} T$ cell proliferation more efficiently than $T_{R E G}$ cells (Fig. 4d-f and Extended Data Fig. 6b), and also reduced their secretion of effector molecules interferon- $\gamma$, Interleukin (IL)-2 and Tumor necrosis factor (TNF) more effectively (Fig. $\mathbf{4 g}$ and Extended Data Fig. 6c). Notably, when compared to $T_{R E G}$ cells, $T_{F R}$ cells reduced the secretion of interferon- $\gamma$ by $\mathrm{CD}^{+} \mathrm{T}$ cells $\sim 4$-fold, and the secretion of IL-2 and TNF by CD8 ${ }^{+} \mathrm{T}$ cells $\sim 2$-fold (Fig. 4h). These data demonstrate that $T_{F R}$ cells are highly suppressive and imply that they are able to actively diminish the effector functions of $C D 8^{+} T$ cells, even at low cell numbers. Based on these results, we chose to transfer OT-I T cells, either alone or with $T_{R E G}$ or $T_{F R}$ cells in a 4:1 ratio, into 
B16F10-OVA tumor-bearing RAG1 KO recipient mice. While the effect of adoptively transferred $T_{R E G}$ cells was negligible, $T_{F R}$ cells substantially inhibited OT-I T cell-mediated tumor rejection (Fig. 4i), demonstrating that $\mathrm{T}_{\mathrm{FR}}$ cells exhibit superior suppressive potential when compared to $T_{R E G}$ cells.

\section{Intratumoral $\mathrm{T}_{\mathrm{FR}}$ cells increase over time}

To further characterize the properties of intratumoral $T_{R E G}$ and $T_{F R}$ cells, we barcoded tumor-infiltrating FOXP3-expressing $\mathrm{CD}^{+} \mathrm{T}$ cells from individual B16F10-OVA tumor-bearing FOXP3-RFP reporter mice from an early (day 11) and a late (day 18) tumor developmental stage, and subjected them to 10x-based single-cell RNA-sequencing. UMAP analysis identified 5 clusters (Fig. 5a) with distinct transcriptomic signatures (Fig. 5b), implying the existence of multiple $T_{\text {REG }}$ cell subsets within tumor tissues. Importantly, all clusters were present at both time points, but only cells in cluster 2 were enriched in d18 (later time point) tumor samples (Fig. 5a,c). GSEA showed that cells in cluster 2 were significantly enriched for signatures linked to $T$ cell activation (Fig. $5 d$ ), $T_{F H}$ and $T_{F R}$ cells (Fig. 5e), and hence depict activated $T_{F R}$ cells, suggesting that $T_{F R}$ cells increase in tumors over time. Single-cell differential gene expression analysis highlighted profound differences in the transcriptome of $T_{F R}$ cells (cluster 2) and $T_{R E G}$ cells in the other clusters (Fig. 5f). In line with our previous data (Extended Data Fig. 2d,e), $T_{F R}$ cells exhibited increased expression of $T_{F R}$ signature genes (i.e. Pdcd1, Tnfrsf18), genes involved in TCR signaling (Cd3g, Cd3d, Cd3e, Lck) as well as of several genes which are

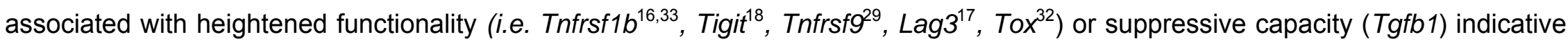
of an activated phenotype and further suggestive of their suppressive potential. $T_{F R}$ cells also showed a significant decrease in the expression of genes that encode receptors required for tissue egress (S1pr1, Klf2), suggesting that they may possess greater tumorresidency properties compared to other $\mathrm{T}_{\mathrm{REG}}$ subsets. (Fig. 5f,g). As in our previous analyses (Extended Data Fig. 5a,c), we found 

cells in cluster 0 , which exhibit features of naïve recirculating $T_{R E G}$ cells (Fig. $\mathbf{5 g}$ ), to $T_{F R}$ cells (Fig. 5i), further corroborating our previous data (Extended Data Fig. 4a and Extended Data Fig. 5d). Together, these data, besides highlighting the transcriptional properties of $T_{F R}$ cells, establish a clonal and developmental relationship between $T_{R E G}$ and $T_{F R}$ cells and are further indicative of intratumoral $T_{R E G}$ to $T_{F R}$ conversion. Based on these findings, we performed a time course experiment which confirmed that the proportion of intratumoral $T_{F R}$ cells, but not $T_{R E G}$ cells, increased with tumor progression (Extended Data Fig. 6d), likely reflective of ongoing $T_{R E G}$ to $T_{F R}$ conversion and higher proliferative potential of $T_{F R}$ cells. Murine tumor-infiltrating $T_{F R}$ cells also showed higher expression of CTLA-4 and PD-1 when compared to $\mathrm{T}_{\mathrm{REG}}$ cells (Fig. 5j), implying that such murine tumor models would be appropriate to test the hypothesis that anti-PD1 therapy increases the numbers and/or function of highly suppressive $T_{F R}$ cells, inducing a profoundly immunosuppressive tumor milieu. Since PD-1 ${ }^{-/-}$mice exhibit increased levels of $\mathrm{T}_{\mathrm{FR}}$ cells in secondary lymphoid organs ${ }^{5}$, we reasoned that PD-1 signaling is likely to restrain expansion of $\mathrm{T}_{\mathrm{FR}}$ cells.

\section{$T_{F R}$ cells are responsive to anti-PD-1 therapy}

Anti-PD-1 monotherapy resulted in a significant increase in the frequency of $\mathrm{T}_{\mathrm{FR}}$ cells in both MC38 and B16F10 tumor models (Fig. 6a and Table 11), suggesting that tumor-infiltrating $T_{R E G}$ (and $T_{F R}$ cells) are highly responsive to blockade of PD-1 signaling, potentially reducing their activation threshold and thus facilitating increased proliferation and differentiation into $\mathrm{T}_{\mathrm{FR}}$ cells. By re-analyzing published single-cell RNA-seq data from patients receiving anti-PD-1 therapy, we found that tumor-infiltrating $T_{F R}$

234 cells from post-treatment samples compared to pre-treatment samples were enriched for transcripts linked to T cell activation and costimulation (Extended Data Fig. 7a and Table 12). Together, these data suggest that engagement of suppressive $T_{F R}$ cells by anti- 
PD1 therapy is likely to diminish its anti-tumor efficacy. To uncouple the effects of $T_{R E G}$ and $T_{F R}$ cells on anti-tumor immunity and anti-

237 PD-1 treatment efficacy, we utilized a genetic knockdown system, in which $\mathrm{T}_{\mathrm{FR}}$ cells can be selectively depleted. Tamoxifen-induced 238 depletion of $T_{F R}$ cells in female heterozygous Foxp $3^{\text {eGFP-cre-ERT2cre/wt }} \times B c / 6^{f / f l}$ mice ${ }^{34}$ prior to initiation of anti-PD-1 therapy, significantly 239 decreased tumor growth, demonstrating that $\mathrm{T}_{\mathrm{FR}}$ cells curtail anti-PD-1 treatment efficacy (Extended Data Fig. 7b). In this system, 240 half of the FOXP3 ${ }^{+}$cells should express the Foxp $3^{\text {eGF-cre-ERT2 }}$ allele (eGFP ${ }^{+}$and BCL-6-deficient) due to random $\mathrm{X}$ chromosome 241 inactivation. Surprisingly however, while we found a decrease in $T_{\mathrm{FR}}$ cells (Extended Data Fig. 7c), we observed a near total loss of 242 BCL-6-deficient eGFP ${ }^{+} T_{R E G}$ cells in the TME (Extended Data Fig. 7d), while the frequency of FOXP3-expressing cells only 243 decreased slightly Extended Data Fig. 7e). These findings were further corroborated in a control experiment by assessing tumor 244 growth and cell frequencies in homozygous Foxp3 $3^{\text {eGFP-cre-ERT2 }}$ and heterozygous Foxp3 ${ }^{\text {eGFP-cre-ERT2/wt }}$ (BCL-6-sufficient) mice 245 (Extended Data Fig. 7f-i). Crucially, these data imply that even a partial $\mathrm{T}_{\mathrm{FR}}$ cell depletion decreases tumor growth, and that BCL-6 expression in $\mathrm{FOXP3}^{+}$cells is likely to be required for their intratumoral persistence. These findings raise several distinct conclusions explaining for the accumulation of eGFP ${ }^{-} \mathrm{FOXP}^{+}$cells; (i) BCL-6-deficiency affects trafficking of $\mathrm{T}_{\mathrm{REG}}$ cells into tumor tissue. (ii) lack of BCL-6 expression precludes adoption of a tissue-residency program, and (iii) BCL-6-deficient $\mathrm{T}_{\mathrm{REG}}$ cells are being outcompeted by their BCL-6-sufficient counterparts. While we cannot formally rule out any of these possibilities, the latter hypothesis is supported by our prior observations of increased proliferative potential, as well as higher expression of TOX and TCF-1 by BCL- $6^{+} \mathrm{T}_{\mathrm{FR}}$ cells 251 compared to BCL-6- $\mathrm{T}_{\mathrm{REG}}$ cells.

To assess the functional importance of $T_{F R}$ cells in tumor development and to further corroborate that $T_{F R}$ cells impair anti-PD-

2531 treatment efficacy, we utilized $T_{F R}$ cell-deficient Foxp $3^{\mathrm{YFP}-c r e} \times B c / 6^{\mathrm{fl/fI}}$ mice $^{35}$. We chose to test this hypothesis in the B16F10-OVA 
melanoma model as it is known to be refractory to anti-PD-1 therapy ${ }^{36}$. Accordingly, anti-PD-1 treatment in control mice did not impact tumor growth (Fig. 6b). Conversely, in $\mathrm{T}_{\mathrm{FR}}$ knockout mice, we found a trend towards lower tumor volume (isotype control), which was significantly reduced by anti-PD-1 therapy (Fig. 6b), demonstrating that $T_{F R}$ cells inhibit the efficacy of anti-PD-1 immunotherapy. To further explore potential impacts of a lack of $T_{F R}$ cells on anti-tumor immunity in this setting, we also assessed tumor-draining axillary and inguinal lymph nodes. The frequency and proliferative capacity of CD8 ${ }^{+}$and $C D 4^{+} \mathrm{T}$ cells in tdLN were similar between the treatment groups (Extended Data Fig. 8a,b). However, akin to our previous findings demonstrating that $\mathrm{T}_{\mathrm{FR}}$ cells can inhibit $\mathrm{CD}^{+} \mathrm{T}$ cell activity and cytokine secretion (Fig.4 g,h), we found that $\mathrm{T}_{\mathrm{FR}}$ cell deficiency results in increased granzyme B expression in $\mathrm{CD}^{+} \mathrm{T}$ cells (Fig. 6c). Together, these data suggest that $\mathrm{T}_{\mathrm{FR}}$ cells inhibit $\mathrm{CD} 8^{+} \mathrm{T}$ cell activity in tumor-draining lymph nodes.

To experimentally validate that $\mathrm{T}_{\mathrm{FR}}$ cells have greater in vivo persistence in tumor tissue, we performed a competition assay, where we co-transferred FOXP3 ${ }^{+} \mathrm{eGFP}^{+}$cells from Foxp3 ${ }^{\mathrm{eGFP}}$ mice (capable of producing $\mathrm{BCL}-6$ ) and $\mathrm{FOXP}^{+} \mathrm{YFP}^{+}$cells from Foxp3 ${ }^{\text {YFP-cre }} \times B c / 6^{\text {fl/fl }}$ mice (incapable of producing BCL-6) in a 1:1 ratio. Strikingly, FOXP3 ${ }^{+} \mathrm{YFP}^{+}$cells failed to accumulate in the spleen and TME (Fig. 6d) of B16F10-OVA tumor-bearing Rag $1^{-1-}$ mice, demonstrating that FOXP3 ${ }^{+} \mathrm{BCL}-6^{+}$cells $\left(\mathrm{T}_{\mathrm{FR}}\right.$ cells) are better suited to survive in the TME. Importantly, the transferred tumor-infiltrating FOXP3 ${ }^{+}$cells expressed significantly higher levels of BCL6 when compared to pre-transfer levels, indicative of $T_{R E G}$ to $T_{F R}$ conversion either inside or outside of the tumor (Fig. 6e), corroborating our previous findings (Extended Data Fig. 5a-c). As it is contentious whether the Foxp3-YFPcre allele should be considered hypomorphic ${ }^{37,38}$, which might account at least partially for the observed phenotype, we performed a control experiment with FOXP3 ${ }^{+} \mathrm{YFP}^{+}$cells from Foxp3 ${ }^{\text {YFP-cre }}$ mice (no Bcl6 floxed allele). In this control setting, we found that FOXP3 ${ }^{+} \mathrm{YFP}^{+}$cells did 
accumulate in spleen and TME, albeit at slightly lower levels than FOXP3 ${ }^{+} \mathrm{RFP}^{+}$cells from Foxp $3^{\mathrm{RFP}}$ mice (Fig. 6f). While these data imply that the knock-in of the YFP-Cre fusion protein might impact FOXP3 expression or cell functionality, they importantly verify that BCL-6 expression is required for the persistence of FOXP3-expressing cells in tumor.

\section{Sequential ICB is beneficial in melanoma patients}

As our findings demonstrated that $\mathrm{T}_{\mathrm{FR}}$ cells curtail the efficacy of anti-PD-1 therapy (Fig. 6b and Extended Data Fig. 7b), we reasoned that it may be necessary to deplete $T_{F R}$ cells in the tumor prior to initiating anti-PD1 therapy to overcome the suppressive milieu induced by anti-PD-1-mediated increase in $T_{F R}$ cells. Anti-CTLA-4 treatment is believed to deplete intratumoral $T_{R E G}$ cells via antibody-dependent cellular cytotoxicity ${ }^{39}$. Given that tumor-infiltrating $T_{F R}$ cells expressed higher levels of CTLA-4 than $T_{R E G}$ cells in both human and mouse, we hypothesized that $\mathrm{T}_{\mathrm{FR}}$ cells should be more efficiently depleted. Indeed, anti-CTLA-4 monotherapy resulted in greater depletion of $T_{F R}$ cells compared to $T_{R E G}$ cells (Extended Data Fig. 8c). These data also indicate that immunotherapy drugs elicit immediate effects on target cell populations and rapidly re-shape the cellular composition within the TME. Based on these results and our previous findings, we reasoned that sequential immune checkpoint blockade (ICB) treatment, where $\mathrm{T}_{\mathrm{FR}}$ cells are initially depleted by anti-CTLA-4, might prove beneficial, as subsequent anti-PD-1 therapy would not activate suppressive cellular targets ( $T_{F R}$ cells) but would instead engage CD8 ${ }^{+}$TILs to enhance anti-tumor immune responses. As before, we tested this in the B16F10-OVA melanoma model, which is refractory to anti-PD-1 therapy ${ }^{36}$. As expected, monotherapy with either anti-CTLA-4 or anti-PD-1 antibodies did not impact tumor growth, whereas depletion of $\mathrm{T}_{\mathrm{FR}}$ cells with anti-CTLA4 followed by antiPD1 therapy led to a significantly reduced tumor volume (Fig. 7a). Consistent with our hypothesis, we found that anti-PD-1 therapy 
increasingly acts on, and hence elevates the frequency of $\mathrm{CD}^{+}$TILs after $\mathrm{T}_{\mathrm{FR}}$ cells have been depleted by anti-CTLA-4 treatment,

291 and also led to an increase in the frequency of granzyme $\mathrm{B}^{+} \mathrm{CD} 8^{+}$and $\mathrm{CD} 4^{+} \mathrm{CTLs}$ (Fig. $7 \mathbf{b}$ ).

To test the clinical significance of sequential ICB treatment, we retrospectively assessed the survival outcomes of patients 293 with inoperable melanoma $(n=271)$, who were, based on their treatment regimens, stratified into 5 groups: $1^{\text {st }}$ line anti-CTLA-4, $1^{\text {st }}$ 294 line anti-PD-1, simultaneous combination therapy, sequential therapy with anti-CTLA-4 followed by anti-PD-1 at progression and vice versa. Sequential treatment with anti-CTLA-4 followed by anti-PD-1 was associated with better long-term overall survival (OS) outcomes when compared to the 4 other groups $(\mathrm{P}<0.001)$ (Fig. 7c and Table 13). It has to be noted though that patients receiving simultaneous ICB therapy exhibited a more advanced disease prior to treatment initiation (higher proportion with AJCC 8 stage M1c and M1d $(n=75)$ than patients on $1^{\text {st }}$ line anti-PD-1 $(n=70)$ or $1^{\text {st }}$ line anti-CTLA-4 $(n=52)$, (Fig. 7d,e and Table 13), likely contributing to their poor OS outcomes. However, the advantageous effect of anti-CTLA-4 followed by anti-PD-1 therapy was preserved in patients with $\mathrm{M} 1 \mathrm{a} / \mathrm{b}$ and $\mathrm{M} 1 \mathrm{c} / \mathrm{d}$, respectively (Fig. 7d,e), indicating that this treatment regimen is clinically beneficial even in patients with very poor prognosis. Differences in BRAF status did not affect ICB treatment outcomes (Fig. 7f). Our outcome data for patients receiving $1^{\text {st }}$ line anti-CTLA-4 appear to be superior to those in a recently published study ${ }^{40}$, but are however not directly comparable, as the proportions of patients going on to receive $2^{\text {nd }}$ line anti-PD-1 treatment was significantly lower in that trial $(43 \%$ vs $63 \%$ ). significantly better survival outcomes ( $P=0.0003)$ (Fig. 7c-e). 
In an independent cohort of patients with melanoma $(n=29)$, who received anti-PD1 treatment, we observed poor survival 308 outcomes in patients with a higher proportion of $\mathrm{CD}^{+} \mathrm{T}$ cells co-expressing FOXP3 and BCL-6 (BCL-6 ${ }^{+} \mathrm{T}_{\mathrm{FR}}$ cells) in tumor 309 (Extended Data Fig. 8d), and also noticed a trend towards a higher frequency of $\mathrm{T}_{\mathrm{FR}}$ cells $\left(\mathrm{BCL} 6^{+} \mathrm{FOXP} 3^{+} \mathrm{CD} 4^{+} \mathrm{T}\right.$ cells) in nonresponders compared to responders to anti-PD-1 treatment (Extended Data Fig. 8e). A lower frequency of CXCR5 ${ }^{+}$cells (Extended

311 Data Fig. 8f), a surrogate marker for the abundance of TLS, was also associated with poor survival outcomes (Extended Data Fig.

$312 \mathbf{8 g}$ ), consistent with recently published studies ${ }^{11-13}$. Given that $T_{F R}$ cells have been shown to mitigate germinal center responses in 313 secondary lymphoid organs, it is tempting to assume that $\mathrm{T}_{\mathrm{FR}}$ cells might not only impede anti-tumor immunity by inhibiting CD8 ${ }^{+}$

314 TILs, but also by regulating TLS in tumor tissues, which should be investigated in future studies.

315

316

\section{Discussion}

$T_{R E G}$ cells impede anti-tumor immunity and are thus detrimental to patient survival. In non-cancer settings, $T_{R E G}$ cells have been shown to differentiate into PD-1 expressing $T_{F R}$ cells that restrain germinal center responses by suppressing GC B cells and stimulatory $T_{F H}$ cells $s^{2,41}$. Both, $T_{F H}$ and $T_{F R}$ cells, constitutively express high levels of the co-inhibitory receptors PD-1, yet few studies have investigated their impact on anti-tumor immunity and their responsiveness to anti-PD-1 therapy. While recent studies have demonstrated that $T_{F H}$ cells, B cells and TLS are associated with patient survival and responsiveness to immunotherapy ${ }^{11-14}$, the precise mechanism, potential cell-cell interactions, drivers and regulators of TLS formation or maintenance, and the importance of specific $B$ and $T$ cell subsets remain unknown.

Here, we provide the first in-depth analysis of tumor-infiltrating $T_{F R}$ cells and elucidate their responsiveness to ICB and the context in which they exert their suppressive functions. $T_{F R}$ cells were prevalent in tumor tissues of several cancer types and 
exhibited superior suppressive capacity and in vivo persistence when compared to $T_{R E G}$ cells, with whom they shared a clonal and developmental relationship. This developmental relationship between intratumoral $T_{R E G}$ and $T_{F R}$ cells was characterized by substantial TCR sharing and a gradual increase of $T_{F R}$ cells over time, which, together with the single cell trajectory analyses and adoptive transfer studies, implies ongoing $T_{R E G}$ to $T_{F R}$ conversion. Crucially, unlike $T_{R E G}$ cells, $T_{F R}$ cells were preferentially located within TLS, and among tumor-infiltrating lymphocytes, $T_{F R}$ cells expressed the highest levels of CTLA-4 and PD-1. Given that $T_{F R}$

331 cells mitigate germinal center responses in secondary lymphoid organs ${ }^{2,41}$, our finding that $T_{F R}$ cells are enriched in TLS suggests

332 that intratumoral $T_{F R}$ cells might also regulate TLS formation and maintenance, potentially by controlling $B$ cell or $T_{F H}$ cell responses,

333 which should be tested in future studies.

334 Our findings in murine tumor models indicate that intratumoral $T_{F R}$ cells are responsive to ICB and that by increasing the 335 abundance of $\mathrm{T}_{\mathrm{FR}}$ cells, anti-PD-1 therapy can not only facilitate, but also dampen anti-tumor immune attack. We provide critical insights into how anti-CTLA-4 and anti-PD-1 therapies mediate their function, and highlight the clinical benefit of sequential dosing to render tumors responsive to anti-PD1 therapy, a hypothesis that merits further investigation in a randomized clinical trial. The welldescribed clinical scenario in which some tumors hyper-progress following anti-PD1 therapy ${ }^{42,43}$ may be explained by the effects of treatment on a highly suppressive immune cell compartment ( $T_{F R}$ cells), especially in patients with an initially high level of tumorspecific $T_{R E G}\left(T_{F R}\right.$ precursor) cells. Conversely, exacerbated immune-related adverse events observed upon combination therapy,

341 might be caused by anti-CTLA-4-mediated depletion or impairment of the activity of FOXP3-expressing cells in multiple tissues and 342 subsequent uninhibited anti-PD-1-mediated activation of effector $\mathrm{CD} 4^{+}$and $\mathrm{CD} 8^{+} \mathrm{T}$ cells, hypotheses which can be addressed in 343 future studies. Finally, our results implicate the unique composition of stimulatory versus suppressive T cells in the TME of each 


\section{References}

356 1. De Simone, M. et al. Transcriptional Landscape of Human Tissue Lymphocytes Unveils Uniqueness of Tumor-Infiltrating T Regulatory Cells. Immunity 45, 1135-1147 (2016).

2. Linterman, M. A. et al. Foxp3+ follicular regulatory T cells control the germinal center response. Nat. Med. 17, 975-982 (2011).

3. Sage, P. T., Paterson, A. M., Lovitch, S. B. \& Sharpe, A. H. The coinhibitory receptor CTLA-4 controls B cell responses by modulating T follicular helper, T follicular regulatory, and T regulatory cells. Immunity 41, 1026-1039 (2014).

4. Sage, P. T., Alvarez, D., Godec, J., Von Andrian, U. H. \& Sharpe, A. H. Circulating T follicular regulatory and helper cells have memory-like properties. J. Clin. Invest. 124, 5191-5204 (2014).

5. Sage, P. T., Francisco, L. M., Carman, C. V. \& Sharpe, A. H. The receptor PD-1 controls follicular regulatory T cells in the lymph nodes and blood. Nat. Immunol. 14, 152-161 (2013). 
6. Vanderleyden, I. et al. Follicular Regulatory T Cells Can Access the Germinal Center Independently of CXCR5. Cell Rep. 30, 611-619.e4 (2020).

7. Brenna, E. et al. CD4+ T Follicular Helper Cells in Human Tonsils and Blood Are Clonally Convergent but Divergent from NonTfh CD4+ Cells. Cell Rep. 30, 137-152.e5 (2020).

8. Romão, V. C. et al. Human blood T fr cells are indicators of ongoing humoral activity not fully licensed with suppressive function . Sci. Immunol. 2, eaan1487 (2017).

9. Ritvo, P.-G. G. et al. $\mathrm{T}_{\mathrm{fr}}$ cells lack IL-2Ra but express decoy IL-1R2 and IL-1Ra and suppress the IL-1-dependent activation of $\mathrm{T}_{\text {fh }}$ cells. Sci. Immunol. 2, eaan0368 (2017).

10. Botta, D. et al. Dynamic regulation of $\mathrm{T}$ follicular regulatory cell responses by interleukin 2 during influenza infection. Nat. Immunol. 18, 1249-1260 (2017).

11. Helmink, B. A. et al. B cells and tertiary lymphoid structures promote immunotherapy response. Nature 577, 549-555 (2020)

12. Cabrita, R. et al. Tertiary lymphoid structures improve immunotherapy and survival in melanoma. Nature 577, 561-565 (2020)

13. Petitprez, F. et al. B cells are associated with survival and immunotherapy response in sarcoma. Nature 577, 556-560 (2020)

14. Hollern, D. P. et al. B Cells and T Follicular Helper Cells Mediate Response to Checkpoint Inhibitors in High Mutation Burden Mouse Models of Breast Cancer. Cell, 179(5):1191-1206.e21 (2019)

15. Chung, Y. et al. Follicular regulatory T cells expressing Foxp3 and Bcl-6 suppress germinal center reactions. Nat Med, 18(8):983-8.

16. Chen, X. et al. Cutting edge: expression of TNFR2 defines a maximally suppressive subset of mouse CD4+CD25+FoxP3+ T regulatory cells: applicability to tumor-infiltrating T regulatory cells. J. Immunol. 180, 6467-6471 (2008).

17. Huang, C.-T. et al. Role of LAG-3 in regulatory T cells. Immunity 21, 503-513 (2004).

18. Joller, N. et al. Treg cells expressing the coinhibitory molecule TIGIT selectively inhibit proinflammatory Th1 and Th17 cell responses. Immunity 40, 569-581 (2014).

19. Hayatsu, N. et al. Analyses of a Mutant Foxp3 Allele Reveal BATF as a Critical Transcription Factor in the Differentiation and Accumulation of Tissue Regulatory T Cells. Immunity 47, 268-283.e9 (2017).

20. Guo, X. et al. Global characterization of T cells in non-small-cell lung cancer by single-cell sequencing. Nat Med. 24, 978-985 (2018).

21. Plitas, G. et al. Regulatory T Cells Exhibit Distinct Features in Human Breast Cancer. Immunity 45, 1122-1134 (2016).

22. Miller, B. C. et al. Subsets of exhausted CD8 + T cells differentially mediate tumor control and respond to checkpoint blockade. Nat. Immunol. 20, 326-336 (2019). 
23. Im, S. J. et al. Defining CD8+ T cells that provide the proliferative burst after PD-1 therapy. Nature 537, 417-421 (2016).

24. Utzschneider, D. T. et al. T Cell Factor 1-Expressing Memory-like CD8+ T Cells Sustain the Immune Response to Chronic Viral Infections. Immunity 45, 415-427 (2016).

25. Siddiqui, I. et al. Intratumoral Tcf1+PD-1+CD8+ T Cells with Stem-like Properties Promote Tumor Control in Response to Vaccination and Checkpoint Blockade Immunotherapy. Immunity 50, 1-17 (2019).

26. Sade-Feldman, M. et al. Defining T Cell States Associated with Response to Checkpoint Immunotherapy in Melanoma. Cell 175, 998-1013.e20 (2018).

27. Ahmadzadeh, M. et al. Tumor-infiltrating human CD4+ regulatory T cells display a distinct TCR repertoire and exhibit tumor and neoantigen reactivity. Sci. Immunol. 4, eaao4310 (2019).

28. $\mathrm{Xu}, \mathrm{L}$. et al. The Kinase mTORC1 Promotes the Generation and Suppressive Function of Follicular Regulatory $\mathrm{T}$ Cells. Immunity 47, 538-551.e5 (2017).

29. Kniemeyer, O., Brakhage, A. A., Ferreira, F., Wallner, M. \& Sawitzki, B. Regulatory T Cell Specificity Directs Tolerance versus Allergy against Aeroantigens in Humans. Cell 167, 1067-1078.e16 (2016).

30. Szabo, P. A., Miron, M. \& Farber, D. L. Location, location, location: Tissue resident memory T cells in mice and humans. Sci. Immunol. 4, eaas9673 (2019).

31. Page, N. et al. Expression of the DNA-Binding Factor TOX Promotes the Encephalitogenic Potential of Microbe-Induced Autoreactive CD8+ T Cells. Immunity 48, 937-950.e8 (2018).

32. Scott, A. C. et al. TOX is a critical regulator of tumour-specific T cell differentiation. Nature 571, 270-274 (2019)

33. Tam, E. M. et al. Antibody-mediated targeting of TNFR2 activates CD8+ T cells in mice and promotes antitumor immunity. Sci. Transl. Med., 11(512):eaax0720 (2019)

34. Fu, W. et al. Deficiency in T follicular regulatory cells promotes autoimmunity. J. Exp. Med. 215, 815-825 (2018).

35. $\mathrm{Wu}, \mathrm{H}$. et al. Follicular regulatory $\mathrm{T}$ cells repress cytokine production by follicular helper $\mathrm{T}$ cells and optimize IgG responses in mice. Eur. J. Immunol. 46, 1152-1161 (2016).

36. Kleffel, S. et al. Melanoma Cell-Intrinsic PD-1 Receptor Functions Promote Tumor Growth. Cell 162, 1242-1256 (2015).

37. Zhang, R. et al. An obligate cell-intrinsic function for CD28 in Tregs. J. Clin. Invest., 123(2):580-93 (2013)

38. Franckaert, D. et al. Promiscuous Foxp3-cre activity reveals a differential requirement for CD28 in Foxp3 + and Foxp3-T cells. Immunol. Cell Biol., 93(4):417-23 (2015)

39. Simpson, T. R. et al. Fc-dependent depletion of tumor-infiltrating regulatory T cells co-defines the efficacy of anti-CTLA-4 therapy against melanoma. J. Exp. Med. 210, 1695-1710 (2013). 
431

40. Wolchok, J. D. et al. Overall Survival with Combined Nivolumab and Ipilimumab in Advanced Melanoma. N. Engl. J. Med., 377(14):1345-1356 (2017).

41. Sage, P. T. et al. Suppression by T FR cells leads to durable and selective inhibition of B cell effector function. Nat. Immunol. 17, 1436-1446 (2016).

42. Champiat, S. et al. Hyperprogressive disease is a new pattern of progression in cancer patients treated by anti-PD-1/PD-L1. Clin. Cancer Res. 23, 1920-1928 (2017).

43. Knorr, D. A. \& Ravetch, J. V. Immunotherapy and hyperprogression: Unwanted outcomes, unclear mechanism. Clin. Cancer Res. 25, 904-906 (2019).

\section{Acknowledgements}

We thank L. Chudley, K. McCann, O. Wood, M. Chamberlain, K. Amer, D. Jeffrey, M. Lane, C. Fixmer, M. Lopez, N. Graham, M. Machado, T. Mellows and B. Johnson for assistance with recruitment of study subjects and processing of samples. We thank Dr. Matthew Wheater for the access to the clinical data from the joint practice with I.K and C.H.O. We thank A. Upadhye for contributions to experimental work. We thank J.B. Lilley for his help on the data collection and analysis of the survival cohort. We recognize C. Kim, D. Hinz and C. Dillingham for their assistance with cell sorting, FACSAria Fusion Cell Sorter - S10 RR027366; S. Liang, A. Wang and $\mathrm{H}$. Simon for assistance with library preparation, next generation sequencing using Illumina HiSeq 2500 - NIH \#S10OD016262 and NovaSeq6000 \#S100D025052-01. Members of the Vijayanand laboratory for their assistance with editing the figures and manuscript. We thank J. Linden and S.Fuchs for providing B16F10-OVA and MC38-OVA tumor cell lines, respectively. Supported by the Wessex Clinical Research Network and the National Institute of Health Research, UK (sample collection), the William K. Bowes Jr Foundation (P.V.), Whittaker foundation (T.S.-E. and C.H.O.), the Cancer Research UK Centres Network Accelerator Award Grant - A21998 (T.S.-E. and C.H.O.), the Faculty of Medicine of the University of Southampton (T.S.-E. and 
445 publish or preparation of the manuscript.

\section{Author Contributions}

447 S.E., J.C., P.S.F., T.S-E., F.A., C.H.O., and P.V., conceived of the work; S.E., J.C., performed experiments. S.E., J.C., B.P., C.R-S, 448 and A.M., analyzed data under the supervision of F.A., C.H.O, P.V.; C.J.H., performed the immunohistochemistry analyses under 449 supervision of G.J.T.; A.A., E.W., S.J.C., I.K., and S.E. assisted in patient recruitment, obtaining consent and sample collection; S.E 450 wrote the first draft of the manuscript that was revised and edited by P.S.F., F.A., C.H.O., and P.V.

451

\section{Declaration of Interests}

453 The authors declare no competing interests.

454

\section{Figure Legends}

456 Figure 1. Tumor-infiltrating $\mathbf{T}_{\mathrm{FR}}$ cells are highly prevalent in human cancers. a, Integrated analysis of 9 single-cell RNA-seq 457 datasets displayed by uniform manifold approximation and projection (UMAP) from 6 different cancer types. Seurat clustering of $45825,149 \mathrm{CD}^{+} \mathrm{T}$ cells colored based on cluster type (left panel) and study (middle panel); Right panel shows Seurat-normalized 459 expression of FOXP3 in different clusters (see also Fig. S1 and Methods). b,c, Bar charts depicting the frequency of FOXP3- and $460 \mathrm{FOXP3}^{+}$or $\mathrm{T}_{\mathrm{REG}}$ in tumor-infiltrating $C D 4^{+} \mathrm{T}$ cells (b), or $B C L 6^{+} \mathrm{T}_{\mathrm{FR}}, C X C R 5^{+} \mathrm{T}_{\mathrm{FR}}, B C L 6^{+} C X C R 5^{+} \mathrm{T}_{\mathrm{FR}}$ in tumor-infiltrating $\mathrm{T}_{\mathrm{REG}}$ cells (c) 461 in the assessed datasets. 
Figure 2. Tumor-infiltrating $T_{F R}$ cells are primarily located in TLS. a-d, flow cytometric analysis and representative contour plots of $C D 4^{+} T$ cells, $T_{R E G}$ cells, $T_{F H}$ cells, $T_{F R}$ cells, and histogram plots of $C D 8^{+}$and $C D 4^{+}$TILs from $n=10$ treatment naïve NSCLC patients depicting the frequency of $\mathrm{CD} 8^{+} \mathrm{T}$ cells (blue, $\mathrm{LIN}^{-} \mathrm{CD} 45^{+} \mathrm{CD} 3^{+} \mathrm{CD} 8^{+}$), $\mathrm{T}_{\mathrm{REG}}$ (teal, $\mathrm{LIN}^{-} \mathrm{CD} 45^{+} \mathrm{CD} 3^{+} \mathrm{CD} 4^{+} \mathrm{CXCR} 5^{-} \mathrm{CD} 127^{-}$ CD25 $5^{+}$, $T_{\mathrm{FH}}$ (light green, $\mathrm{LIN}^{-} \mathrm{CD} 45^{+} \mathrm{CD} 3^{+} \mathrm{CD} 4^{+} \mathrm{CXCR} 5^{+} \mathrm{GITR}$ ) and $\mathrm{T}_{\mathrm{FR}}$ (yellow, $\mathrm{LIN}^{-} \mathrm{CD} 45^{+} \mathrm{CD} 3^{+} \mathrm{CD} 4^{+} \mathrm{CXCR} 5^{+} \mathrm{GITR}{ }^{+}$) cells $(\mathbf{a})$, the frequency and mean fluorescence intensity (MFI) of CD25 and ICOS (\%CD25, P=0,002; MFI CD25, P=0.0137; \%ICOS, P=0.0645, MFI ICOS, $P=0.0039$ for indicated comparisons) (b) intracellular CTLA-4 expression and MFI in $T_{\text {REG }}$ (teal, LIN ${ }^{-}$ $\mathrm{CD}^{+} 5^{+} \mathrm{CD}^{+} \mathrm{CD}^{+} \mathrm{CXCR} 5^{-} \mathrm{FOXP3}{ }^{+} \mathrm{BCL}-6^{-}$), $\quad \mathrm{T}_{\mathrm{FH}}$ (light green, $\mathrm{LIN}^{-} \mathrm{CD} 45^{+} \mathrm{CD} 3^{+} \mathrm{CD} 4^{+} \mathrm{BCL}-6^{+} \mathrm{FOXP3} 3^{-}$) and $\mathrm{T}_{\mathrm{FR}}$ (yellow, LIN $\left.\mathrm{CD} 5^{+} \mathrm{CD3}^{+} \mathrm{CD}^{+} \mathrm{BCL}-6^{+} \mathrm{FOXP}^{+}\right)(\% \mathrm{CTLA}-4, \mathrm{P}=0.0039$, MFI CTLA-4, $\mathrm{P}=0.0020$ for indicated comparisons) (c), and the frequency and MFI of PD-1 expression (\%PD-1, P=0.0020; MFI PD-1, P=0.0020 for indicated comparisons) (d), grey depicts respective 471 fluorescence minus one (FMO) controls in histogram plots. e, Whole-slide multiplexed immunohistochemistry analysis of $T_{\text {REG }}$ and $472 T_{F R}$ cells in NSCLC tissue sections from patients in a-d, Micrographs show PanCK (white), CD4 (light blue), CXCR5 (yellow), CD20 473 (magenta) FOXP3 (green) and BCL-6 (red), pink arrows characterize CD4 ${ }^{+} \mathrm{FOXP3}{ }^{+} \mathrm{BCL}-6^{+} \mathrm{T}_{\mathrm{FR}}$ cells in a region of interest selected 474 for high density of $\mathrm{T}_{F R}$ cells. Bar graphs show the proportion of FOXP3 ${ }^{-}$and FOXP3 ${ }^{+} \mathrm{CD}^{+}$cells (upper panel) or $\mathrm{T}_{\mathrm{REG}}$ and $\mathrm{T}_{\mathrm{FR}}$ cells 475 (lower panel) from whole-slide histo-cytometry analyses of each sample as in (a-d), scale bars are $250 \mu \mathrm{m}$ (left panel) and $25 \mu \mathrm{m}$ 476 (right panel). f, Proportion of $\mathrm{T}_{\mathrm{REG}}$ (teal) and $\mathrm{T}_{\mathrm{FR}}$ (yellow) cells in tumor stroma versus tertiary lymphoid structures (TLS) ( $\mathrm{P}=0.0002$ $477\left(T_{R E G}\right)$ and $P=0.0002\left(T_{F R}\right)$ from whole-slide histo-cytometry analyses for $n=8$ treatment naïve NSCLC patients. All data are mean $+/-$ 478 S.E.M.; Two-tailed Wilcoxon matched-pairs signed rank test between $T_{R E G}$ and $T_{F R}$ cells (b-d) and two-tailed Mann-Whitney test 479 between TLS and stroma localization for $T_{R E G}$ and $T_{F R}$ cells (f). 
481 Figure 3. Comparison of human tumor-infiltrating $T_{R E G}$ and $T_{F R}$ cells. a, Analysis of 10x single-cell RNA-seq data displayed by 482 manifold approximation and projection (UMAP). Seurat clustering of $8,722 \mathrm{CD}^{+}$and $\mathrm{CD} 8^{+} \mathrm{T}$ cells from primary tumor tissue and 483 metastasized tumor-infiltrated lymph nodes colored based on cluster type (left panel), the other three panels are showing Seurat484 normalized expression of $C D 8 B, C D 4$ and FOXP3 respectively. b, Heatmap comparing gene expression of cells in cluster 1 versus 485 cluster 6 . Depicted are transcripts that change in expression more than 0.25 -fold and adjusted $P$ value of $\leq 0.05$. c,d Gene set 486 enrichment analysis for follicular feature ${ }^{44}$ (c) and $T_{F R}$ feature genes (d), derived from Fig. 3j) for cells in cluster 6 and cluster 1 487 ordered by Log2 fold change. e, Ingenuity pathway analysis of differentially expressed transcripts $(n=1245)$ between cluster 1 and 488 cluster 6. f, Violin plots comparing expression levels of indicated transcripts in cluster 1 (left) and cluster 6 (right) cells. g, TraCer 489 plots of all clonally expanded cells (=/>2 clonotypes) in cluster 1 and cluster 6 colored by cluster origin (cluster 1 green, cluster 6 490 yellow). h, Euler diagram shows overlap between clonotypes in cluster 1 and cluster 6 . i, bar chart depicting the mean percentage of 491 clonally expanded cells in cluster 1 and cluster 6. j, Heatmap illustrating the intersection of differentially expressed genes (with mean

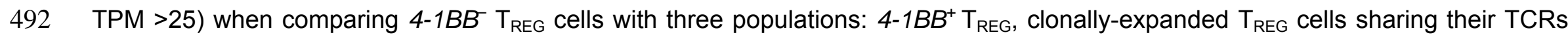
493 with $T_{F R}$ and clonally-expanded $T_{F R}$ cells (distinct cell populations are indicated with colored bars). Genes linked to 494 immunosuppressive function, co-stimulation, and tissue residency are highlighted.

495

496 Figure 4. Frequency and functional responsiveness of $T_{F R}$ cells in murine tumor models. a-c, Mice were inoculated with 497 B16F10-OVA or MC38-OVA cells subcutaneously (s.c.) on the right flank. Analyses of tumor-infiltrating $\mathrm{T}_{\mathrm{REG}}$ (CD19- 
$\left.\mathrm{CD} 45^{+} \mathrm{CD} 3^{+} \mathrm{CD} 4^{+} \mathrm{BCL}-6{ }^{-} \mathrm{FOXP} 3^{+}\right)$and $\mathrm{T}_{\mathrm{FR}}\left(\mathrm{CD} 19^{-} \mathrm{CD} 45^{+} \mathrm{CD} 3^{+} \mathrm{CD} 4^{+} \mathrm{BCL}-6^{+} \mathrm{FOXP} 3^{+}\right)$cells were performed, a, Flow-cytometric analysis of the frequency of tumor-infiltrating $T_{R E G}$ and $T_{F R}$ cells in indicated tumor models at day 21 after tumor inoculation ( $n=6$ mice for B16F10 and n=7 mice for MC38). b, Flow-cytometric analysis of the MFI and frequencies of expression of $\mathrm{KI}-67(\mathrm{P}=0.002)$, TCF-1 $(P=0.002)$ and 4-1BB (0.002) in indicated cell types in the B16F10-OVA model at day14 after tumor inoculation ( $n=10$ mice/group). $\mathbf{c}$ Representative FACS plots depicting the expression of TOX (x-axis) and TCF-1 (y-axis) in CD8 ${ }^{+} T_{\text {cells, }} T_{R E G}$ cells and $T_{F R}$ cells, Flow-cytometric analysis of the frequency of TOX-expressing cells in indicated cell types in the B16F10-OVA model at day14 ( $\mathrm{n}=10$ mice/group). d, Mice were immunized intraperitoneally (i.p.) with Ovalbumin in alum and treated with an IL-2/anti-IL2R complex at days 3,4 and 5 for in vivo $\mathrm{T}_{\mathrm{REG}}$ cell expansion; shown are representative FACS plots characterizing splenic $\mathrm{T}_{\mathrm{REG}}\left(\mathrm{CD} 4^{+} \mathrm{CXCR} 5^{-}\right.$ $\left.\mathrm{CD}^{2} 5^{+} \mathrm{GITR}^{+}\right)$and $\mathrm{T}_{\mathrm{FR}}\left(\mathrm{CD} 4^{+} \mathrm{CXCR} 5^{+} \mathrm{CD} 25^{+} \mathrm{GITR}^{+}\right)$cells, e, Representative histogram plots depicting the dilution of cell trace violet (CTV) in $\mathrm{CD}^{+} \mathrm{T}$ cells with or without addition of $\mathrm{T}_{\mathrm{REG}}$ or $\mathrm{T}_{\mathrm{FR}}$ cells. $\mathbf{f}$, Flow-cytometric analysis of an in vitro proliferation assay showing the frequency of proliferating $\mathrm{CD}^{+} \mathrm{T}$ cells when co-cultured with different proportions of $\mathrm{T}_{\mathrm{REG}}$ cells (green) or $\mathrm{T}_{\mathrm{FR}}$ cells (yellow), depicted are the results for $n=3$ technical replicates for the dilutions and $n=4$ technical replicates for $C D 8^{+} T$ cells $(1: 0$ dilution). $\mathbf{g}$, Luminex analysis of supernatants from an in vitro proliferation assay $(\mathbf{e}, \mathbf{f})$, depicted is the concentration of secreted IFN- $\gamma$, IL-2 and TNF for $n=2$ technical replicates. $\mathbf{h}$, fold-change reduction in secretion of indicated cytokines between $T_{R E G}$ and $T_{F R}$ cells at a $4: 1$ ratio of CD8 $T$ cells to either $T_{R E G}$ or $T_{F R}$ cells. $i$, indicated cells were transferred into B16F10-OVA tumor-bearing RAG1 ${ }^{-/-}$recipient mice at day 3 after tumor inoculation, tumor volume of mice treated as indicated is shown ( $\mathrm{n}=5$ mice/group). Data are mean $+/-$ S.E.M.; Significance for comparisons were computed using two-tailed Mann-Whitney test. All data are representative of two independent experiments. 
517 Figure 5. Intratumoral $\mathrm{T}_{\mathrm{FR}}$ cells gradually increase over time. a, Analysis of 10x single-cell RNA-seq data displayed by uniform manifold approximation and projection (UMAP). Seurat clustering of tumor-infiltrating FOXP3-expressing T cells colored based on cluster type, the panels shows UMAPs of tumor-infiltrating FOXP3-expressing T cells at d11 (left panel) and d18 (right panel). Charts show proportion of cells in individual mice for the indicated time point. Percentages for cells in cluster 2 ( $T_{F R}$ cells) are depicted. b, Heatmap showing genes enriched in the identified clusters. Depicted are transcripts that significant change in expression (> 2-fold and adjusted $P$ value of $\leq 0.05$.) c, Bar charts depicting the proportion of cells in each cluster, colored based on tumor developmental stage (d11 versus d18). d,e, Gene set enrichment analysis for a T cell activation signature

$(\mathbf{d}), \mathrm{T}_{\mathrm{FH}}$ signatures $^{44}(\mathbf{e})$, derived from versus the other clusters ordered by Log2 fold change. f, Volcano plot of cells in cluster 2 versus the other clusters, depicted are differentially expressed transcripts (adjusted $P$ value of $\leq 0.05$ ) that change in expression more than 2 -fold. $\mathbf{g}$, Plot shows average transcript expression (color scale) and percent of expressing cells (size scale) for selected genes in each cluster. $\mathbf{h}$, Euler diagram shows overlap between clonotypes in cluster 2 and the other clusters. i, Single-cell pseudo-time trajectory analysis of tumorinfiltrating FOXP3-expressing T cells (a) constructed using the Monocle3 algorithm. j, Flow-cytometric analysis depicting the MFI of the expression of PD-1 (P=0.002) and CTLA-4 (P=0.002) in indicated cell types in the B16F10-OVA model at day14 after tumor

531 inoculation. Representative histogram plots are displayed. Data in (j) are mean +/- S.E.M.; Significance for comparisons were computed using two-tailed Wilcoxon matched-pairs signed rank test. Data in (j) are representative of two independent experiments. 
as fold induction of both cell types following anti-PD-1 therapy in the B16F10-OVA model (left panel, n=9 mice/group) and MC38-

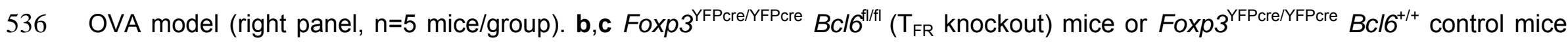
537 were s.c. inoculated with B16F10-OVA cells and treated with isotype control or anti-PD-1 Abs at indicated time points, Tumor volume

538 (b) for $n=7-9$ mice/group and frequency of granzyme $B^{+} C D 8^{+} T$ cells in tumor-draining lymph nodes (c) of mice treated as indicated in

539 (b), $n=7-9$ mice/group. d, Mice were immunized i.p. with Ovalbumin in alum and additionally treated with an IL-2/anti-IL-2R complex 540 at days 3,4 and 5. OT-I CD8 ${ }^{+}$T cells, GFP ${ }^{+}$and $\mathrm{YFP}^{+} \mathrm{T}_{\mathrm{REG}}$ cells were adoptively transferred into B16F10-OVA tumor-bearing RAG1

541 I' mice at day 3 after tumor inoculation. In $n=7$ mice, the frequencies of eGFP and YFP cells in spleen (left, $P=0.007$ ) or tumor tissue

542 (right, $\mathrm{P}=0.0006$ ) are shown. e, Flow-cytometric analysis of $\mathrm{BCL}-6$ expression in splenic CD4 ${ }^{+} \mathrm{FOXP} 3^{+}$cells of Foxp3 $3^{\mathrm{YFP}-\mathrm{cre}} \times \mathrm{BC}^{\mathrm{ff} / \mathrm{fl}}$ 543 mice (grey), Foxp3 ${ }^{\mathrm{eGFP}}$ mice (blue) and tumor-infiltrating CD4 ${ }^{+} \mathrm{FOXP} 3^{+}$cells (red) 13 days after adoptive transfer into B16F10-OVA 544 tumor-bearing RAG1 KO mice. f, Mice were immunized i.p. with Ovalbumin in alum and additionally treated with an IL-2/anti-IL-2R 545 complex at days 3,4 and 5 . OT-I CD8 ${ }^{+} \mathrm{T}$ cells, $\mathrm{RFP}^{+}$and $\mathrm{YFP}^{+} \mathrm{T}_{\mathrm{REG}}$ cells were adoptively transferred into B16F10-OVA tumor546 bearing Rag1 KO mice at day 3 after tumor inoculation. In $\mathrm{n}=6$ mice/group, the frequencies of $\mathrm{RFP}^{+}$and $\mathrm{YFP}^{+}$in spleen (left, $547 \mathrm{P}=0.0087$ ) or tumor tissue (right, $\mathrm{P}=0.0022$ ) are shown. Data are mean +/- S.E.M.; Significance for comparisons were computed 548 using two-tailed Mann-Whitney test $(\mathbf{a}, \mathbf{b}, \mathbf{d}, \mathbf{f})$ or one-way ANOVA comparing the mean of each group with the mean of the control group (ctrl+anti-PD-1) followed by Dunnett's test (c). All data are representative of two independent experiments.

551 Figure 7. Clinical benefit of sequential ICB. a-c, Mice were s.c. inoculated with B16F10-OVA cells and treated with anti-CTLA-4 552 (day 10 and day 13, n=8 mice), anti-PD-1 (day 14 and day 17, n=10 mice), anti-CTLA-4 (day 10 and day 13) and anti-PD-1 Abs (day 55314 and day17) $(n=8$ mice) or isotype treated control mice $(n=13$ mice) at indicated time points, tumor volume (a) and cell frequencies 
(b) of mice treated as indicated. (P-value style for $\left.\mathbf{a}, \mathbf{b}=0.1234(\mathrm{~ns}), 0.0332\left({ }^{*}\right), 0.0021\left(^{* *}\right), 0.0002\left({ }^{* * *}\right),<0.0001\left({ }^{* * * *}\right)\right) \mathbf{c}$, Survival curves of an independent cohort of melanoma patients $(n=271)$ stratified into 5 groups based on ICB treatment regimen. $\mathbf{d}$,e, Survival curves for patients stratified into those with early onset disease (d) (M1a and M1b combined), late stage disease (e) (M1c and M1d combined) or BRAF mutation status (f) of patient cohort. Two-tailed Mann-Whitney test (a), one-way ANOVA was used to compare the mean of each group with the mean of the control group (B16F10) followed by Dunnett's test (b) or Mantel-Cox test (c-e). Data in a,b are mean +/- S.E.M and are representative of two independent experiments.

\section{Methods}

Human tumor samples. The study was approved by the Southampton and South West Hampshire Research Ethics Board (ethics committee MREC number 14/SC/0186, NIHR portfolio adoption ID 16818), and written informed consent was obtained from all subjects. Newly diagnosed, untreated patients with NSCLC (or HNSCC), were prospectively recruited once referred. Freshly resected tumor tissue was obtained from lung cancer patients following surgical resection and after histological confirmation. The patient cohort for the survival analysis was collected by retrospective evaluation of a centralized prescribing system (Aria, Varian Medical Systems Inc). All patients started on immunotherapy at a single institution (Southampton University Hospitals NHS Foundation Trust) with immunotherapy for melanoma between 07/2014 to 10/2018 were included. Patients were divided into cohorts according to first type immunotherapy treatment approved in the United Kingdom (anti-PD-1, either nivolumab or pembrolizumab, $\mathrm{N}=98$ ), anti-CTLA-4 antibody (ipilimumab (88) or joint administration of nivolumab plus ipilimumab on up to four occasions ( $\mathrm{N}=85$ ), followed by maintenance nivolumab where appropriate. Dosing was according to standard of care at the time (3mg/kg ipilimumab $\mathrm{x}$ $4,2 \mathrm{mg} / \mathrm{kg}$ of pembrolizumab 3 weekly, later $200 \mathrm{mg}$ flat dosing, $3 \mathrm{mg} / \mathrm{kg}$ nivolumab, then $480 \mathrm{mg}$ flat dosing, and in combination 

dose of immunotherapy. Clinical data were obtained from an electronic hospital record for age, gender, BRAF status, LDH, M stage, performance status. For clinical outcome overall survival was collected to death or censored at last clinical review. Data were anonymized by the treating clinician (I.K. and C.H.O.) once the data had been collated and verified. Prism 8 (Graph Pad Software) was used for ANOVA and to plot Kaplan Meier Survival Graphs and estimate treatment differences using a Log-rank (Mantel-Cox) test on survival curves. SPSS v26 (IBM Corp) was used to evaluate imbalances between treatment groups via Chi Square testing followed by Cox Regression analysis. Fur multiple testing a Bonferroni error correction was applied.

Mice. C57BL/6J (JAX stock \#000664), Bc/6 fl/fl (JAX stock \#023727), OT-I (JAX stock \#003831) and RAG1 KO (JAX stock \#002216),

581 Foxp3 $3^{\text {YFP-cre }}$ (JAX stock \#016959) mice were obtained from Jackson labs. Foxp3 ${ }^{\text {eGFP-cre-ERT2 }}$ (JAX stock \#016961) and Foxp3 ${ }^{\text {RFP }}$ (JAX 582 stock \#008374) were a kind gift from K. Ley (LJI) and Foxp3 ${ }^{\text {eGFP }}$ (JAX stok \#006772) mice were a kind gift from A. Altman (LJI). 583 Female mice (age 6-12 weeks) were used for all experiments. The housing temperature in the vivarium is controlled and ranges from $58469-75 F$, humidity is not controlled but monitored and ranges from 30-70\%. The light/dark cycles are from 6am-6pm, respectively. All 585 animal work was approved by the relevant La Jolla institute for Immunology Animal Ethics Committee.

586
Tumor cell lines. B16F10-OVA cells were a gift from the laboratory of J. Linden (LJ) and MC38-OVA cells were a gift from the lab S. Fuchs (UPenn) and approved for use by M. Smyth (Peter MacCallum cancer center). Cell lines tested negative for mycoplasma infection and were subsequently treated with Plasmocin to prevent contamination.

Tumor models. Tumor cell lines were tested negatively for mycoplasma infection and Plasmocin (InvivoGen) was used as a routine addition to culture media to prevent mycoplasma contamination. Mice were inoculated with $1-1.5 \times 10^{5}$ B16F10-OVA cells or $2 \times 10^{6}$ 
MC38-OVA cells subcutaneously into the right flank. Mice were injected intraperitoneally at indicated time points with either 200 $\mu \mathrm{g}$ anti-PD-1 (29F1.A12, InvivoPlus anti-mouse PD-1, Bioxcell), anti-CTLA-4 (9H10, InvivoPlus anti-mouse CTLA-4, Bioxcell) or respective isotype controls (anti-CTLA-4 isotype ctrl, InVivoPlus polyclonal Syrian hamster IgG, Bioxcell) (anti-PD-1 isotype control, InVivoPlus rat IgG2a isotype control, anti-trinitrophenol, Bioxcell). Tumor size was monitored every other day, and tumor harvested at indicated time points for analysis of tumor-infiltrating lymphocytes. Tumor volume was calculated as $1 / 2 \times D \times d^{2}$, where $D$ is the major axis and $\mathrm{d}$ is the minor axis, as described previously ${ }^{45}$. Tumor growth was monitored at least thrice weekly to ensure that tumors did not exceed $25 \mathrm{~mm}$ in diameter.

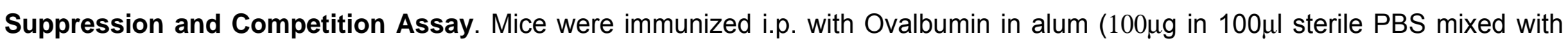

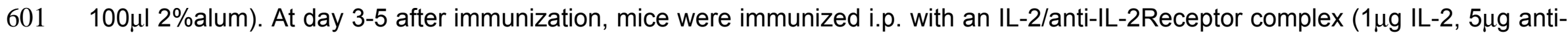
IL-2Receptor $A b$, mixed for $30 \mathrm{~min}$ at $37^{\circ} \mathrm{C}$ ) to achieve polyclonal expansion of $\mathrm{T}_{\mathrm{REG}}$ cells in vivo, as described previously ${ }^{46}$. Lymphocytes $\left(\mathrm{CD}^{+}\right.$and $\mathrm{CD}^{+} \mathrm{T}$ cells) were isolated from spleen by mechanical dispersion through a $70-\mu \mathrm{m}$ cell strainer (Miltenyi) to generate single-cell suspensions. $\mathrm{CD}^{+}$and $\mathrm{CD} 8^{+} \mathrm{T}$ cells were purified (Stemcell) according to manufacturer's instructions.

605 In Vitro $-\mathrm{CD}^{+} \mathrm{T}$ cells were labelled with CellTrace Violet (CTV) (Thermofisher) and 40,000 cells were added to 96 well cell culture 606 plated, pre-coated with anti-CD3, in $200 \mu$ l complete RPMI media. Purified CD4 ${ }^{+} \mathrm{T}_{\text {cells }}$ were stained and different numbers of viable

607 (Fixable Viability dye) $\mathrm{T}_{\text {REG }}$ cells $\left(\mathrm{CD} 4^{+} \mathrm{CXCR} 5^{-} \mathrm{CD} 25^{+} \mathrm{GITR}^{+}\right)$or $\mathrm{T}_{\mathrm{FR}}$ cells $\left(\mathrm{CD} 4^{+} \mathrm{CXCR} 5^{+} \mathrm{CD} 25^{+}\right.$GITR ${ }^{+}$) were sorted into the cell culture 608 plate containing the CTV-labeled $\mathrm{CD}^{+} \mathrm{T}$ cells. $\mathrm{CD}^{+} \mathrm{T}$ cell proliferation (CTV dilution) was determined 3 days later.

609 In Vivo - OT-I CD8 ${ }^{+} \mathrm{T}$ cells were purified (Stemcell), CD4 ${ }^{+} \mathrm{T}$ cells were purified, stained and $\mathrm{T}_{\mathrm{REG}}$ and $\mathrm{T}_{\mathrm{FR}}$ cells were sorted as 610 described above. Cells were counted and $2 \times 10^{5}$ OT-I T cells, $2 \times 10^{5}$ OT-I T cells $+5 \times 10^{4} \mathrm{~T}_{\mathrm{REG}}$ cells $\left(4: 1\right.$ ratio) or $2 \times 10^{5}$ OT-I T cells + 
$6115 \times 10^{4} \mathrm{~T}_{\mathrm{FR}}$ cells (4:1 ratio) were adoptively transferred into B16F10-OVA tumor-bearing RAG1 KO recipient mice 3 days after tumor 612 inoculation.

613 Competition assay - OT-I CD8 ${ }^{+}$T cells were purified (Stemcell), FOXP3 ${ }^{+} \mathrm{T}_{\text {cells were purified from Foxp3 }}^{\mathrm{YFP}-\text { cre }} \times$ Bcl $^{\mathrm{fl/fl}} \mathrm{mice}^{\mathrm{Y}}\left(\mathrm{YFP}{ }^{+}\right.$) 614 and Foxp $3^{\text {eGFP }}$ mice $\left(\mathrm{GFP}^{+}\right)$or from Foxp3 $3^{\text {RFP }}$ mice for the control experiments and $4 \times 10^{5}$ cells $\left(2 \times 10^{5} \mathrm{OT}_{-} \mathrm{I} \mathrm{T}\right.$ cells, $1 \times 10^{5} \mathrm{GFP}^{+}$or

$615 \mathrm{RFP}^{+} \mathrm{T}_{\mathrm{REG}}$ cells and $1 \times 10^{5} \mathrm{YFP}^{+} \mathrm{T}_{\mathrm{REG}}$ cells) were adoptively transferred into B16F10-OVA tumor-bearing RAG1 KO recipient mice 3 616 days after tumor inoculation.

617

618 Flow cytometry. T cells from cryopreserved tumor tissue was mechanically dissociated and digested enzymatically as previously 619 described ${ }^{47}$. Cells were treated with FcR blocking antibody (BD Biosciences) and stained in PBS with $2 \%$ FBS, $2 \mathrm{mM}$ EDTA for 30 620 minutes at $4^{\circ} \mathrm{C}$. For selected markers, secondary stains were performed. Samples were subsequently sorted or fixed for intracellular 621 staining with the FOXP3 TF kit (eBioscience) according to manufacturer's instructions. For all staining, cell viability was verified using 622 fixable viability dye (ThermoFisher).

623 Murine samples - Lymphocytes were isolated from spleen by mechanical dispersion through a 70- $\mu \mathrm{m}$ cell strainer (Miltenyi) to 624 generate single-cell suspensions. RBC lysis (Biolegend) was performed to remove red blood cells. Tumor samples were harvested 625 and lymphocytes were isolated by dispersing the tumor tissue in $2 \mathrm{ml}$ of PBS, followed by incubation of samples at $37^{\circ} \mathrm{C}$ for $15 \mathrm{~min}$ 626 with DNase I (Sigma) and Liberase DL (Roche). Samples were passed through a 70- $\mu$ m cell strainer to create single-cell 627 suspensions. Cells were prepared in staining buffer (PBS with 2\% FBS and 2mM EDTA) and FcR blocked (clone 2.4G2, BD 628 Biosciences) and stained with indicated primary antibodies for 30 minutes at $4^{\circ} \mathrm{C}$; secondary stains were done for selected markers. 629 Samples were then sorted or fixed and intracellularly stained using a FOXP3 transcription factor kit according to manufacturer's 
630 instructions (eBioscience). Cell viability was determined using fixable viability dye (ThermoFisher). For bulk-RNA-seq analyses, we

631 sorted tumor-infiltrating $T_{F R}$ cells based on the co-expression of CXCR5 and GITR ${ }^{2,5}$ (Extended Data Fig. 1b), a surface marker that

632 distinguishes $T_{F H}$ cells from $T_{F R}$ cells. To accurately assess the expression of intracellularly stored molecules like CTLA-4, we

633 characterized $T_{F R}$ cells based on co-expression of BCL-6 and FOXP3 (Extended Data Fig. 1c) since cell fixation led to epitope

634 masking of CXCR5 (Extended Data Fig. 1c, bottom left plot) and GITR. All samples were acquired on a BD FACS Fortessa or

635 sorted on a BD FACS Fusion (both BD Biosciences) and analyzed using FlowJo 10.4.1.

636

637

638 The primary antibodies used for IHC include anti-CD8 (pre-diluted, C8/144B, Agilent Dako), anti-CD4 (1:100, 4B12, Agilent Dako),

639 anti-FOXP3 (1:100, ab20034, Abcam), anti-CXCR5 (1:50, D6L3C, CellSignaling), anti-BCL-6 (1:30, NCL-L-Bcl6-6-564, Leica), anti-

640 CD31 (pre-diluted product diluted further 1:5, Agilent Dako) and anti-PanCK (AE1/AE3; pre-diluted; Agilent Dako). Samples for

641 immunohistochemistry analyses were prepared, stained and analyzed as previously described ${ }^{48}$. Cells were identified by nucleus

642 detection and cytoplasmic regions were simulated up to $5 \mu \mathrm{m}$, per cell protein expression was measured using the mean staining

643 intensity within simulated cell regions.

644

645 Bulk-RNA sequencing. Total RNA was purified using a miRNAeasy kit (Qiagen) from human tumor-infiltrating $T_{\text {REG }}$ (LIN ${ }^{-}$ $\left.646 \mathrm{CD} 45^{+} \mathrm{CD}^{+}{ }^{+} \mathrm{CD} 4^{+} \mathrm{CXCR} 5^{-} \mathrm{CD} 127^{-} \mathrm{CD} 25^{+}\right)$and $\mathrm{T}_{\mathrm{FR}}\left(\mathrm{LIN}^{-} \mathrm{CD} 45^{+} \mathrm{CD} 3^{+} \mathrm{CD} 4^{+} \mathrm{CXCR} 5^{+} \mathrm{GITR}^{+}\right)$cells and was quantified as described 647 previously ${ }^{47,49}$. Cells from mice immunized with either Ovalbumin in Complete Freund's adjuvant (InvivoGen), Ovalbumin in

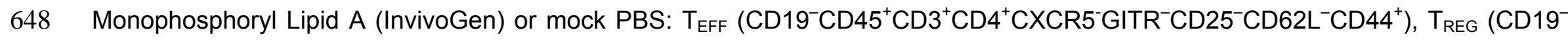
$\left.649 \mathrm{CD} 45^{+} \mathrm{CD}^{+} \mathrm{CD}^{+} \mathrm{CXCR} 5^{-} \mathrm{GITR}^{+} \mathrm{CD} 25^{+}\right), \quad \mathrm{T}_{\mathrm{FH}} \quad\left(\mathrm{CD}_{19}{ }^{-} \mathrm{CD}_{4} 5^{+} \mathrm{CD}^{+} \mathrm{CD}^{+} \mathrm{CXCR}^{+} \mathrm{GITR}^{-}\right) \quad$ and $^{2} \quad \mathrm{~T}_{\mathrm{FR}} \quad\left(\mathrm{CD}_{19}\right.$ 
$\mathrm{CD}_{4}{ }^{+} \mathrm{CD} 3^{+} \mathrm{CD} 4^{+} \mathrm{CXCR} 5^{+} \mathrm{GITR}{ }^{+}$) were sorted and RNA was purified as described above. RNA-seq libraries were prepared using Smart-seq2 protocol and sequenced on Illumina platform, as previously described ${ }^{50}$. Quality control steps were applied as previously described $^{47}$. Samples failing quality controls, or having a low number of cells were excluded from further sequencing and analysis.

Bulk RNA-seq analysis. Bulk RNA-seq data from human samples were mapped against the hg19 reference using TopHat ${ }^{51}$ (-bowtie1 -max-multihits 1 -microexon search) with FastQC (v0.11.2), Bowtie ${ }^{52}$ (v1.1.2), Samtools (v0.1.19.0) ${ }^{53}$ and we employed htseq-count -m union -s no -t exon -i gene_name (part of the HTSeq framework, version v0.7.1) ${ }^{54}$. Trimmomatic (v0.36) was used to remove adapters ${ }^{55}$. Bulk RNA-seq from mouse samples were mapped against mm10 reference using TopHat (1.4.1) with library-type fr-unstranded parameter. Values throughout are displayed as $\log _{2}$ TPM (transcripts per million) counts; a value of 1 was added prior to log transformation. To identify genes expressed differentially by various cell types, we performed negative binomial tests for unpaired comparisons by employing the Bioconductor package DESeq2 ${ }^{56}$ ( 1 1.14.1), disabling the default options for independent filtering and Cooks cutoff. We considered genes to be expressed differentially by any comparison when the DESeq2 analysis resulted in a Benjamini-Hochberg-adjusted $P$ value of $\leq 0.05$ and a fold change of at least 2 . Euler diagrams were generated using the eulerr package (v5.1.0). Correlations and heatmaps were generated as previously described ${ }^{49,57,58}$. Visualizations were generated in ggplot2 using custom scripts. For tSNE analysis, the data frame was filtered to genes with mean $\geq 1$ TPM counts expression in at least one condition and visualizations created using the top 500 most variable genes, as calculated in DESeq2 ${ }^{56}$ (v1.16.1); this allowed for unbiased visualization of the $\log _{2}$ (TPM counts + 1) data, using package Rtsne (v0.13). Data in heatmaps are shown as $\log 2$ normalized z-scores. 
670 the TPM data matrix. Well-expressed genes with TPM >= 10 in at least one sample, were used in both $T_{F R}$ and $T_{R E G}$ data from

671 human. Gene modules were generated using blockwiseModules function (parameters: checkMissingData $=$ TRUE, power $=5$,

672 TOMType $=$ "signed", minModuleSize $=30$, maxBlockSize $=13441$, mergeCutHeight $=0.80)$. The remaining parameters were as per

673 default in WGCNA. The default 'grey' module generated by WGCNA for non-co-expressed genes, was excluded from further

674 analysis. As each module by definition is comprised of highly correlated genes, their combined expression may be usefully

675 summarized by module eigengene (ME) profiles, effectively the first principal component of a given module. A small number of

676 module eigengene profiles may effectively 'summarize' the principle patterns within the transcriptome with minimal loss of

677 information. This dimensionality-reduction approach aids correlation of MEs with clinical traits as a module-trait relationship matrix.

678 Significance of correlation between this trait and MEs was assessed using linear regression with Benjamini-Hochberg adjustment to

679 correct for multiple testing. The TOMplot was generated using the TOMplot function in WGCNA with default parameters for clustering

680 and color scheme. To visualize co-expression networks were generated in gplots (v3.0.1) using the heatmap2 function, while

681 weighted correlation analysis was completed using WGCNA ${ }^{59}$ (v1.61) from the $\log _{2}$ (TPM counts + 1) data matrix and the function

682 TOMsimilarityfromExpr (Beta $=5$ ) and exportNetworkToCytoscape, weighted $=$ true, threshold $=0.05$. Highlighted genes were

683 ordered as per the order in the correlation plot. Networks were generated in Gephi (v0.92) ${ }^{60,61}$ using ForceAtlas2 and Noverlap

684 functions. Color and size were scaled to the Average Degree calculated in Gephi. Edge width was scaled to the WGCNA edge

685 weight value.

686 
687 Meta-analysis of published single-cell RNA-seq studies. We integrated 9 published single-cell RNA-seq datasets ${ }^{20,26,62-68}$ of

688 tumor-infiltrating CD4-expressing T cells with UMAP. The integration was performed using the R package Seurat v3.0. For each

689 dataset, cells that expressed less than 200 genes were considered outliers and discarded. We integrated data from all cohorts using

690 the alignment by 'anchors' option in Seurat 3.0. Briefly, the alignment is a computational strategy to "anchor" diverse datasets

691 together, facilitating the integration and comparison of single cell measurements from different technologies and modalities. The

692 "anchors" correspond to similar biological states between datasets. These pairwise correspondences between datasets allows the

693 transformation of datasets into a shared space regardless of the existence of large technical and/or biological divergences. This

694 improved function in Seurat 3.0 allows integration of multiple RNA-seq datasets generated by different platforms ${ }^{69}$. While we agree

695 that single cell RNA-seq can be utilized to identify distinct states within a given cell population, it does not offer higher resolution

696 compared to bulk RNA-seq in terms of number of transcripts recovered due to high drop-out rates with single-cell RNA-seq assays,

697 more so with 10X-based assays. We used the FindlntegrationAnchors function to find correspondences across the different study

698 datasets with default parameters (dimensionality $=1: 30$ ). Furthermore, we used the IntegrateData function to generate a Seurat

699 Object with an integrated and batch-corrected expression matrix. In total, 25,149 cells and 2,000 most variable genes were used for

700 clustering. We used the standard workflow from Seurat, scaling the integrated data, finding relevant components with PCA and

701 visualizing the results with UMAP. The number of relevant components was determined from an elbow plot. UMAP dimensionality

702 reduction and clustering were applied with the following parameters: 2000 genes, 15 principal components, resolution of 0.2 , min.dis

7030.05 and spread 2. Cells used for the integration were selected from clusters labeled in the original studies as tumor CD4 T cells and

704 from pre-treatment samples when necessary. Cells with expression of CD8B > 1 CPM (UMI data) or 10 TPM (Smart-seq2) were 
filtered out as indicated in Table 1. $T_{R E G}$ cells and $T_{F R}$ cells were identified based on criteria defined in Table 1. Only Smart-seq2 706 datasets were used to compare $T_{\mathrm{FR}}$ cells from different cancer types.

707

Single-cell differential expression analysis. Differential expression was calculated with $\mathrm{MAST}^{70}$ and $\mathrm{SCDE}^{71}$ (V1.99.1) as previously described ${ }^{57}$. For each comparison, we obtained the differentially expressed gene lists by taking the union of the gene lists from both the methods using adjusted $\mathrm{P}<0.05$ and $\log _{2}$ fold change $>1$ from each method.

Single-cell TCR and transcriptome analysis: Single-cell Smart-seq2 data from ${ }^{20}$ were re-analyzed (Table 1), using custom scripts to identify $\alpha \beta$ chains and showing only cells were both TCR chains were detected, as described previously ${ }^{56}$. Visualizations were completed in ggplot2, Prism (v8.1.1) and custom scripts in TraCer. A cell was considered expanded when both the most highly expressed $\alpha$ and $\beta$ TCR chain sequences matched other cells with the same stringent criteria. Cells were considered not expanded when $\alpha$ and $\beta$ TCR productive chain sequences did not match those of any other cells. A cell was considered a $T_{\text {REG }}$ cell when the expression of CD4 and FOXP3 were $>10$ TPM, and lacked expression of CXCR5 and BCL6 (TPM $\leq 10)$. A cell was characterized as a $\mathrm{T}_{\mathrm{FR}}$ cell if expression of CD4 and FOXP3 were > 10 TPM and the expression of CXCR5 or BCL6 was > 10 TPM. A cell was considered $4-1 B B^{+}$when the expression of $4-1 B B$ was $>10$ TPM as indicated in Table 1. Cell-state hierarchy maps were generated using Monocle $(\mathrm{v} 3.0)^{72}$ and default settings with expressionFamily = negbinomial.size () , lowerDetectionLimit $=0.1$ after transformation of TPM counts with relative2abs function as recommended in the manual, including the top 2000 most variable genes identified in Seurat (v3.0) and taking 14 PCs based on the elbow plot. The shared signature was calculated with AddModuleScore function from Seurat after setting the object with default parameters and using the intersection of differentially expressed genes from

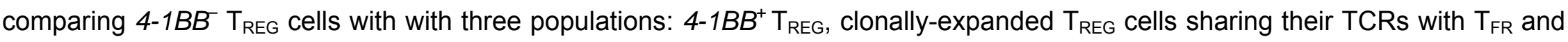


clonally-expanded $T_{F R}$ cells with Benjamini-Hochberg-adjusted $P$ value of $<0.05$ and a log2 fold change of 1 .Single-cell smart-seq2 data from ${ }^{26}$ were utilized to compare the single-cell transcriptome of tumor-infiltrating $T_{F R}$ cells from pre- and post-anti-PD-1 treatment samples. Data in heatmaps are shown as log2 normalized z-scores.

728

729

Hierarchical clustering. Distance between clusters was calculated by obtaining a particular cells location in PCA space (Principal component 1:5) using the function Embeddings from Seurat. The number of principal components was determined from an elbow plot. A distance matrix was calculated (dist function, core R, method = Euclidean) from the PCA matrix and the clustering was performed (hclust function, method="average") in R and generated from the distance matrix. Function colored_bars from the WGCNA package was used to annotate different groups in the dendrogram.

\section{Single-cell transcriptome analysis of primary tumor tissue and metastatic tumor-infiltrated lymph nodes. Human T cells from}

2 HNSCC patients (primary tumor tissue and metastatic tumor-infiltrated lymph nodes) were isolated and prepared as described above. CD4 ${ }^{+} \mathrm{T}_{\mathrm{H}}$ cells (CXCR5 ${ }^{+} \mathrm{GITR}^{-}$and CXCR5 $\left.{ }^{-} \mathrm{CD} 25^{-}\right), \mathrm{T}_{\mathrm{REG}}$ cells $\left.\left(\mathrm{CD} 4^{+} \mathrm{CXCR} 5^{-} \mathrm{CD} 25^{+} \mathrm{CD} 127^{\mathrm{lo}}\right), \mathrm{T}_{\mathrm{FR}} \mathrm{Cells}_{(\mathrm{CD}}{ }^{+} \mathrm{CXCR} 5^{+} \mathrm{GITR}{ }^{+}\right)$ and $\mathrm{CD}^{+} \mathrm{CD}^{+} 9^{+}$cells were sorted and cDNA libraries were constructed using the standard 10x sequencing protocol. A total of $n=9,562$ ( $n=4,975$ from metastatic tumor-infiltrated lymph node, $n=4,589$ from primary tumor tissue) cells were sequenced and cells with less than 200 and more than 5,000 expressed genes, less than 15,000 counts, and more than $10 \%$ of mitochondrial counts were filtered out. For clustering with Seurat (3.0) we used 17 PCs from a set of highly variable genes $(n=609)$ taking $30 \%$ of the variance after filtering out genes with mean expression less than 0.1 and removing TCR genes. TCR analysis: clonotype output (clonotypes and filtered contig annotation) from Cell Ranger for tumor and lymph node libraries were re-calculated (matching sequences were assigned the same clonotype id) and the overlap between cluster 1 and 6 was determined with these 'aggregated' 
tables. Gene Set Enrichment analysis: the Log2 fold change was used as ranking metric and enrichment was calculated for each list.

746 The package fgsea (v1.13.0) in R with default parameters was used to calculate the enrichment and create GSEA plots. Monocle 747 (v2.99.1) was used to generate the trajectory plots, reduction_method = DDRTree for the dimensional reduction taking 15 principal 748 components. Hierarchical clustering was performed as stated above using 20 PCs.

749

Single-cell transcriptome analysis of tumor-infiltrating FOXP3-expressing cells. FOXP3-expressing $\left(\mathrm{RFP}^{+}\right)$cells were isolated 751 from tumor tissues at day 11 or day 18 after B16F10-OVA tumor-inoculation of Foxp3 ${ }^{\text {RFP }}$ reporter mice. Four mice from each time 752 point were barcoded with murine Totalseq-C antibodies (Biolegend). Live/Dead ${ }^{-}$CD $19^{-} \mathrm{CD}^{+} \mathrm{CD}^{+}{ }^{+} \mathrm{RFP}{ }^{+}$cells were sorted and $\mathrm{cDNA}$ 753 libraries were constructed using the standard 10x sequencing protocol. Gene expression, TCR, and antibody capture data was 754 processed with Cell Ranger (v3.1.0). Antibody capture data was analyzed with custom scripts (github.com/vijaybioinfo/ab_capture) 755 as previously described ${ }^{73}$. Differential gene expression analysis was performed as described above. One contaminating cluster 756 exhibiting high expression of transcripts associated with non- $T_{R E G}$ cells (i.e. CD4OLg) was removed prior to differential gene 757 expression analysis. Finally, TCR data was analyzed using custom scripts in R taking clone data for each barcode as indicated in 758 Cell Ranger's output. Euler diagrams and Enrichment plots were generated with eulerr (v6.1.0) and fgsea (v1.10.1), respectively. 759

\section{$760 \quad$ Accession codes}

761 Expression data has been deposited in the Gene Expression Omnibus database under the Super Series 762 Accession Number GSE132297. This Super Series includes data from human and mouse samples.

763

\section{Code availability}


Scripts used for this study are available in our repository on GitHub (https://github.com/vijaybioinfo/TFR_2021). An explanation of each of the is included as well as version changes.

\section{Quantification and statistical analysis}

The number of subjects, samples or mice/group, replication in independent experiments, and statistical tests can be found in the figure legends. Details on quality control, sample elimination and displayed data are stated in the method details and figure legends. Sample sizes were chosen based on published studies to ensure sufficient numbers of mice in each group enabling reliable statistical testing and accounting for variability. Sample sizes are indicated in Figure legends. Mice, which didn't develop any tumors by 10 after inoculation were excluded from analyses, prior to any therapeutic intervention. RNA-seq samples that didn't pass quality control weren't included in the analyses. Experiments were reliably reproduced in independent experiments at least twice. Only female mice were used in the experiments and animals of similar age were randomly assigned to experimental groups. Statistical analyses were performed with Graph Pad Prism 8 and statistical tests used are indicated in the figure legends and experimental model and subject details.

\section{References Methods}

44. Locci, M. et al. Human circulating PD-1+CXCR3-CXCR5+ memory Tfh cells are highly functional and correlate with broadly neutralizing HIV antibody responses. Immunity 39, 758-769 (2013).

45. Juneja, V. R. et al. PD-L1 on tumor cells is sufficient for immune evasion in immunogenic tumors and inhibits CD8 T cell cytotoxicity. J. Exp. Med. 214, 895-904 (2017).

46. Webster, K. E. et al. In vivo expansion of t reg cells with il-2-mab complexes: induction of resistance to eae and long-term acceptance of islet allografts without immunosuppression. J. Exp. Med. 206, 751-760 (2009).

47. Ganesan, A. P. et al. Tissue-resident memory features are linked to the magnitude of cytotoxic T cell responses in human lung 
cancer. Nat. Immunol. 18, 940-950 (2017).

48. Singh, D. et al. CD4+ follicular helper-like T cells are key players in anti-tumor immunity. bioRxiv 2020.01.08.898346 (2020) doi:10.1101/2020.01.08.898346.

49. Engel, I. et al. Innate-like functions of natural killer T cell subsets result from highly divergent gene programs. Nat. Immunol. 17, 728-739 (2016).

51. Trapnell, C., Pachter, L. \& Salzberg, S. L. TopHat: discovering splice junctions with RNA-Seq. Bioinformatics 25, 1105-1111 (2009).

52. Langmead, B., Trapnell, C., Pop, M. \& Salzberg, S. L. Ultrafast and memory-efficient alignment of short DNA sequences to the human genome. Genome Biol. 10, R25 (2009).

53. Li, H. \& Durbin, R. Fast and accurate short read alignment with Burrows-Wheeler transform. Bioinformatics 25, 1754-1760 (2009).

54. Anders, S., Pyl, P. T. \& Huber, W. HTSeq--a Python framework to work with high-throughput sequencing data. Bioinformatics 31, 166-169 (2015).

55. Bolger, A. M., Lohse, M. \& Usadel, B. Trimmomatic: a flexible trimmer for Illumina sequence data. Bioinformatics 30, 21142120 (2014).

56. Love, M. I., Huber, W. \& Anders, S. Moderated estimation of fold change and dispersion for RNA-seq data with DESeq2. Genome Biol. 15, 550 (2014).

57. Patil, V. S. et al. Precursors of human CD4 + cytotoxic T lymphocytes identified by single-cell transcriptome analysis. Sci. Immunol. 3, 8664 (2018).

58. Ganesan, A.-P. et al. Tissue-resident memory features are linked to the magnitude of cytotoxic T cell responses in human lung cancer. Nat. Immunol. 18, 940-950 (2017).

59. Langfelder, P. \& Horvath, S. WGCNA: an R package for weighted correlation network analysis. BMC Bioinformatics 9 , 559 (2008).

60. Mellone, M. et al. Induction of fibroblast senescence generates a non-fibrogenic myofibroblast phenotype that differentially impacts on cancer prognosis. Aging (Albany. NY). 9, 114-132 (2016).

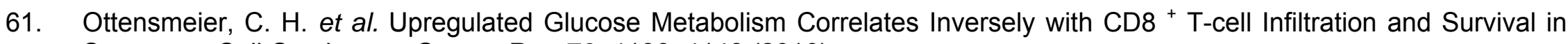
Squamous Cell Carcinoma. Cancer Res. 76, 4136-4148 (2016).

62. Zheng, C. et al. Landscape of Infiltrating T Cells in Liver Cancer Revealed by Single-Cell Sequencing Resource Landscape of 
Infiltrating T Cells in Liver Cancer Revealed by Single-Cell Sequencing. Cell 169, 1342-1356.e16 (2017).

818 63. Zhang, L. et al. Lineage tracking reveals dynamic relationships of T cells in colorectal cancer. Nature 564, 268-272 (2018).

819 64. Savas, P. et al. Single-cell profiling of breast cancer T cells reveals a tissue-resident memory subset associated with improved $820 \quad$ prognosis. Nat. Med. 24, 986-993 (2018).

821 65. Lambrechts, D. et al. Phenotype molding of stromal cells in the lung tumor microenvironment. Nat. Med. 24, 1277-1289 822 (2018).

66. Puram, S. V. et al. Single-Cell Transcriptomic Analysis of Primary and Metastatic Tumor Ecosystems in Head and Neck Cancer. Cell 171, 1611-1624.e24 (2017).

67. Jerby-Arnon, L. et al. A Cancer Cell Program Promotes T Cell Exclusion and Resistance to Checkpoint Blockade. Cell 175, 984-997.e24 (2018).

68. $\mathrm{Li}, \mathrm{H}$. et al. Dysfunctional CD8 T Cells Form a Proliferative, Dynamically Regulated Compartment within Human Melanoma. Cell 176, 775-789.e18 (2019).

69. Stuart, T. et al. Comprehensive Integration of Single-Cell Data. Cell 177, 1888-1902.e21 (2019).

70. Finak, G. et al. MAST: A flexible statistical framework for assessing transcriptional changes and characterizing heterogeneity in single-cell RNA sequencing data. Genome Biol. 16, 278 (2015).

71. Kharchenko, P. V., Silberstein, L. \& Scadden, D. T. Bayesian approach to single-cell differential expression analysis. Nat. Methods 11, 740-742 (2014).

72. Trapnell, C. et al. The dynamics and regulators of cell fate decisions are revealed by pseudotemporal ordering of single cells. Nat. Biotechnol. 32, 381-386 (2014).

73. Meckiff, B. J. et al. Imbalance of Regulatory and Cytotoxic SARS-CoV-2-Reactive CD4+ T Cells in COVID-19. Cell, 183, 1340$1353(2020)$

838 


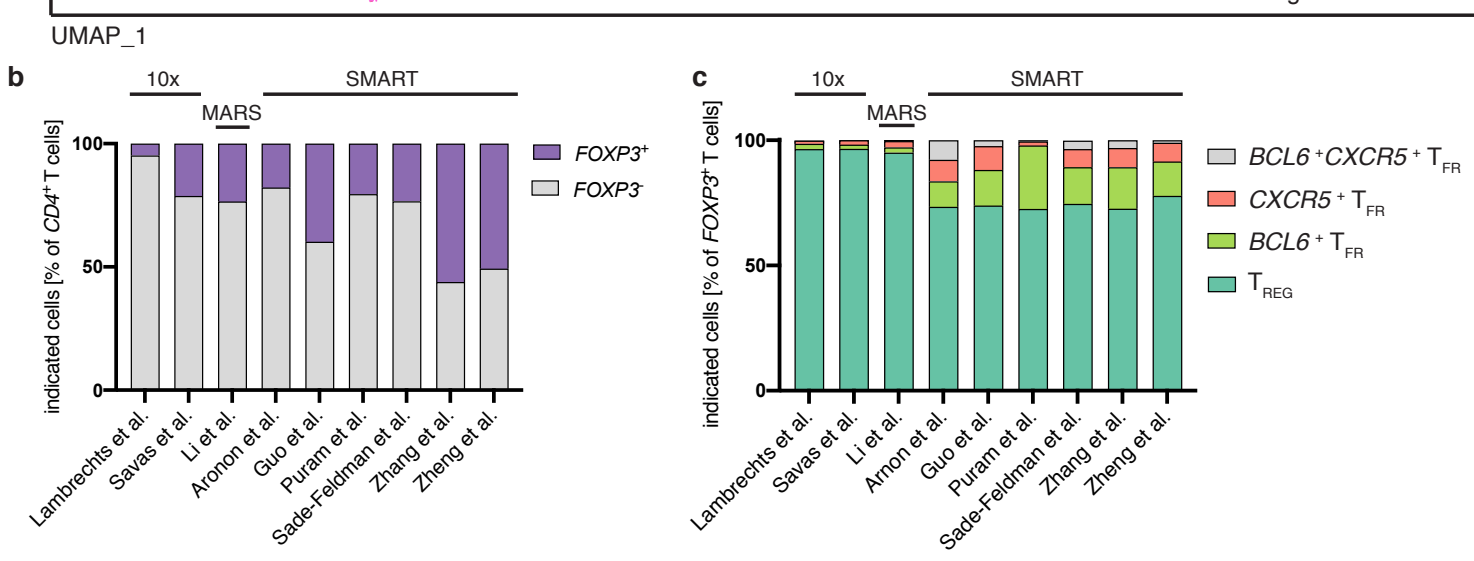




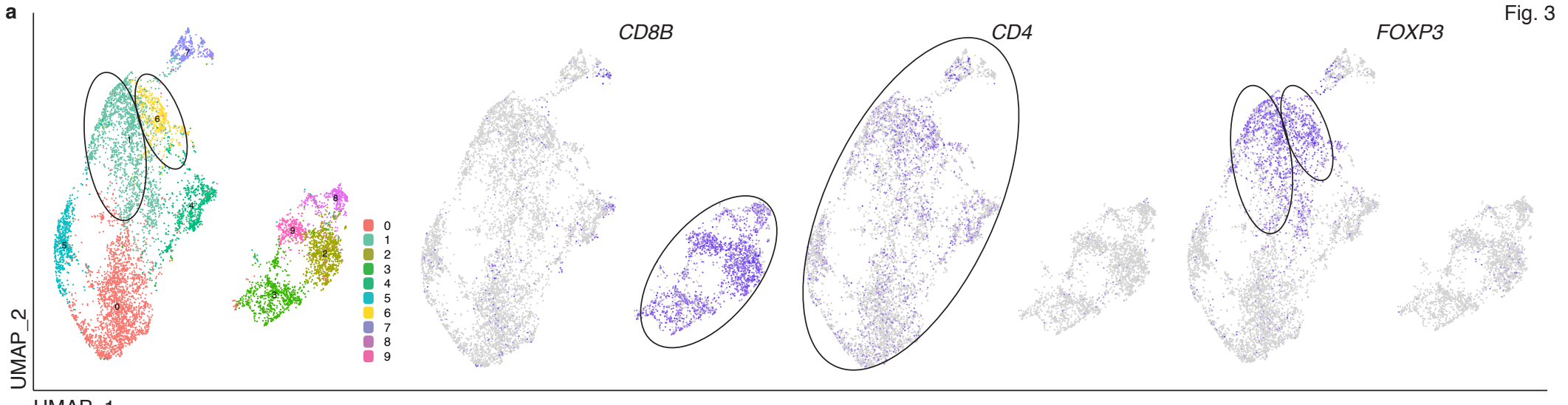

\section{UMAP_1}

b

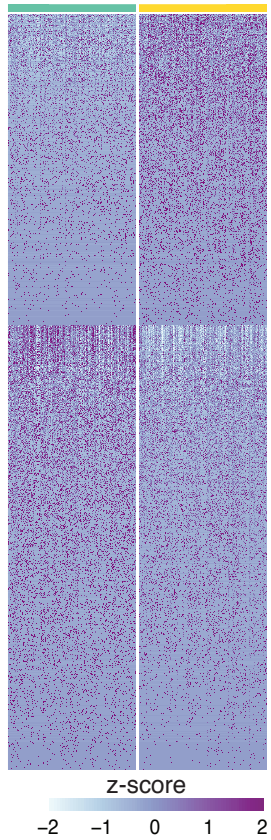

c follicular signature

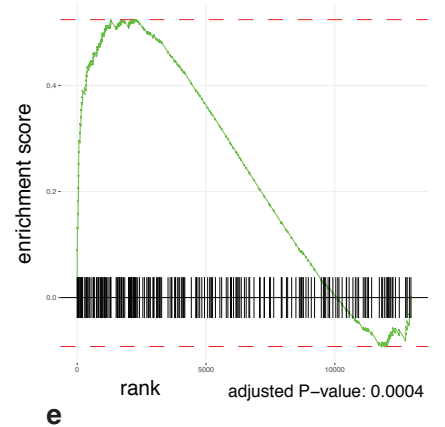

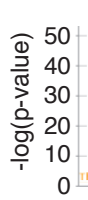

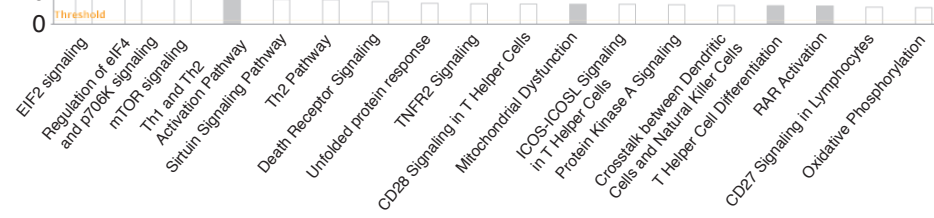

d $\quad \mathrm{T}_{\mathrm{FR}}$ signature

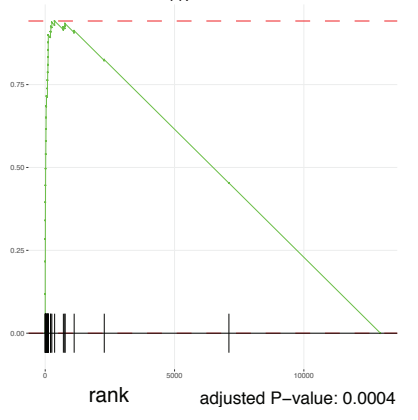

cluster $1 \square$ cluster 6

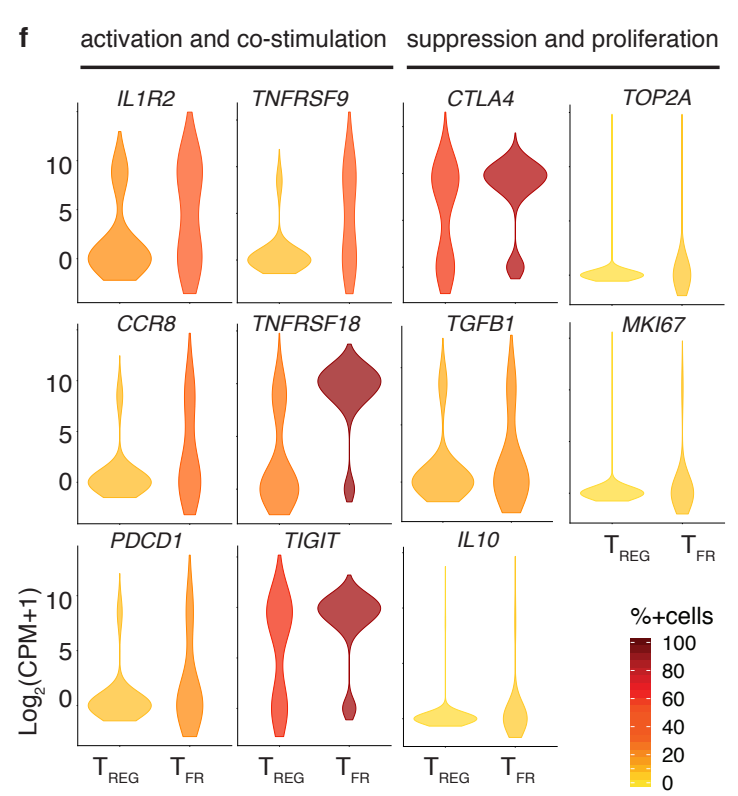

巨

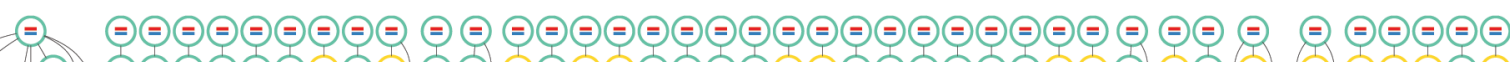

$\Theta \Theta \Theta$ $\Theta$ $=\Theta \Theta \Theta$
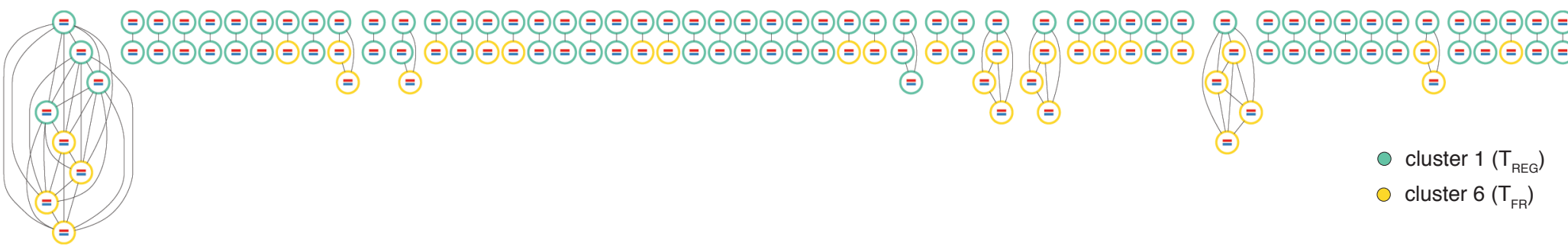

cluster $6\left(T_{F R}\right)$

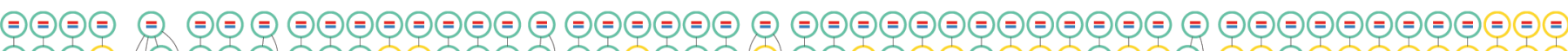

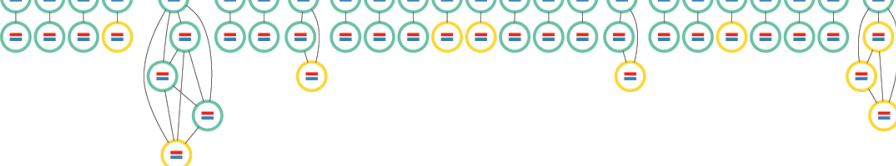
$\lim _{=}=$
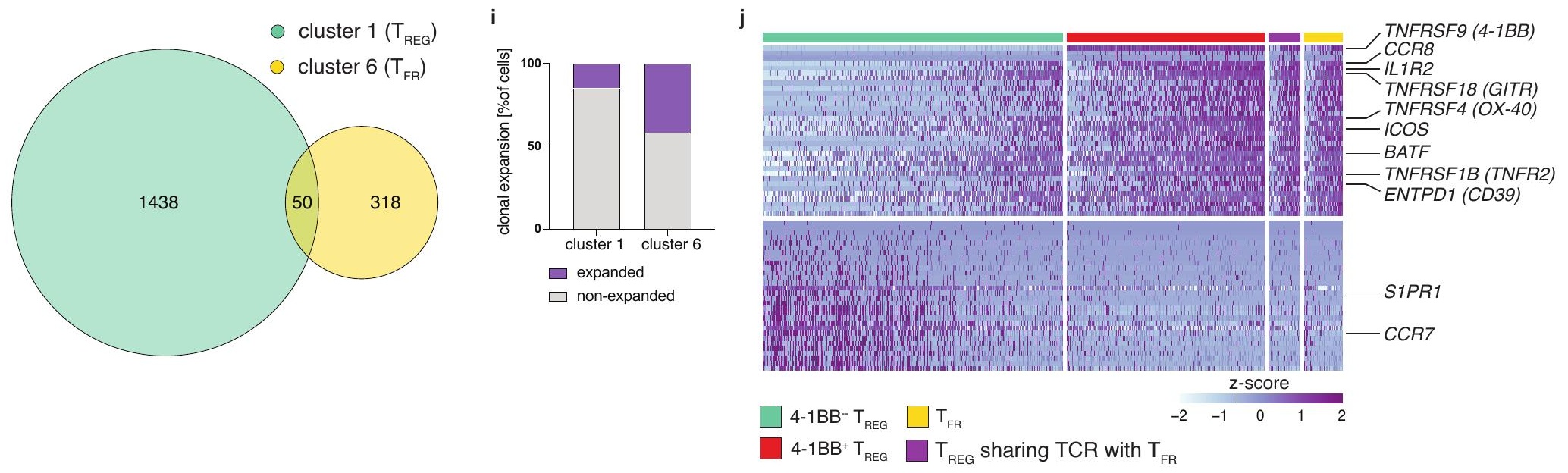


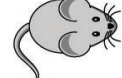

OVA-CFA

OVA-MPLA

PBS
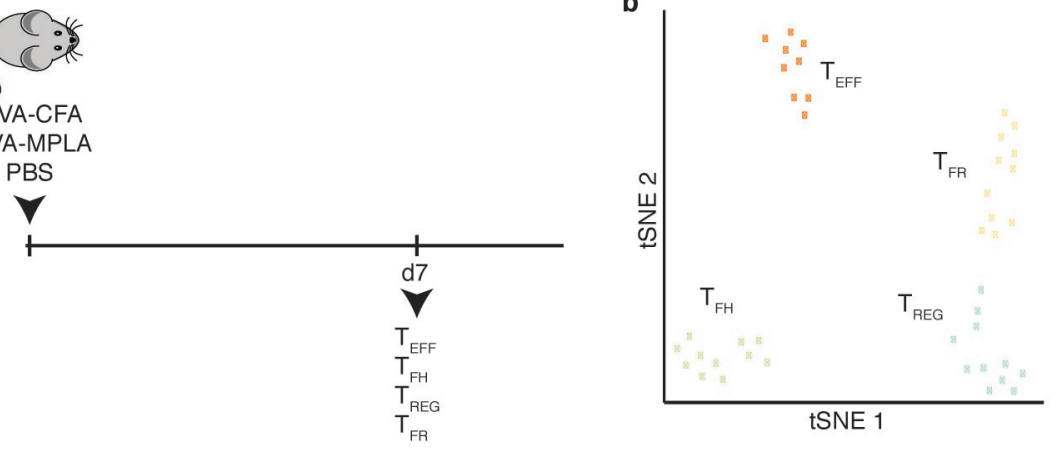

upregulated in $\mathrm{T}_{\mathrm{FP}}$

- $\mathrm{T}_{\mathrm{FR}}$ versus. $\mathrm{T}_{\mathrm{FH}}$ downregulated in $\mathrm{T}_{\mathrm{FR}}$

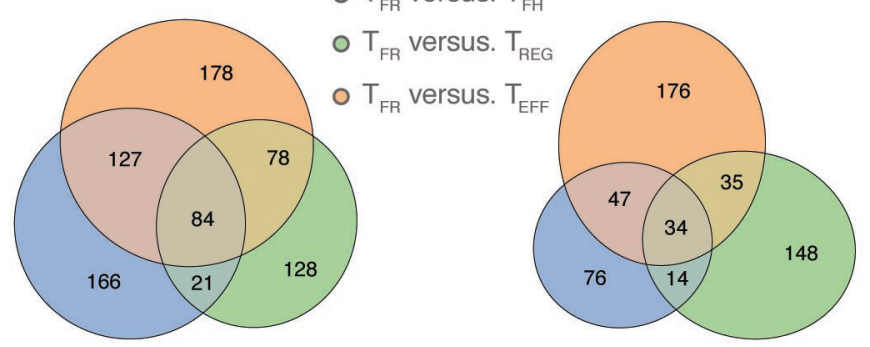

Ascl2

Cxcr5

Bcl6

Maf

$\stackrel{\infty}{\sim}$

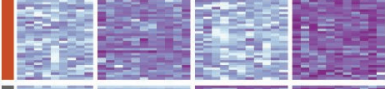

Tnfrsf4 $(0 x-40)$

Ikzf4 (Ikaros)

FoxP3

Ctla 4

Ik2f2 (Helios)

Tnfrsf18 (Gitr)

Prdm1 (Blimp1)

1110

ลิ

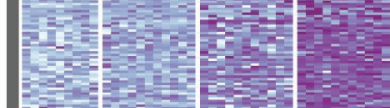

KIf2

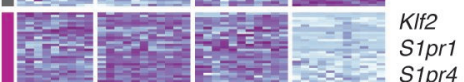

ষ

ले
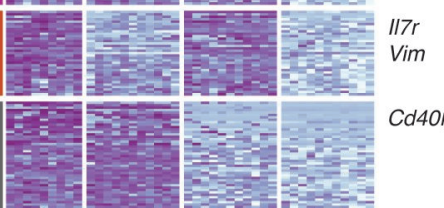

Cd40lg

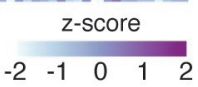

$\mathrm{T}_{\mathrm{EFF}} \square \mathrm{T}_{\text {REG }}$

common

$\square \mathrm{T}_{\mathrm{FH}} \square \mathrm{T}_{\mathrm{FR}}$

follicular

regulatory e
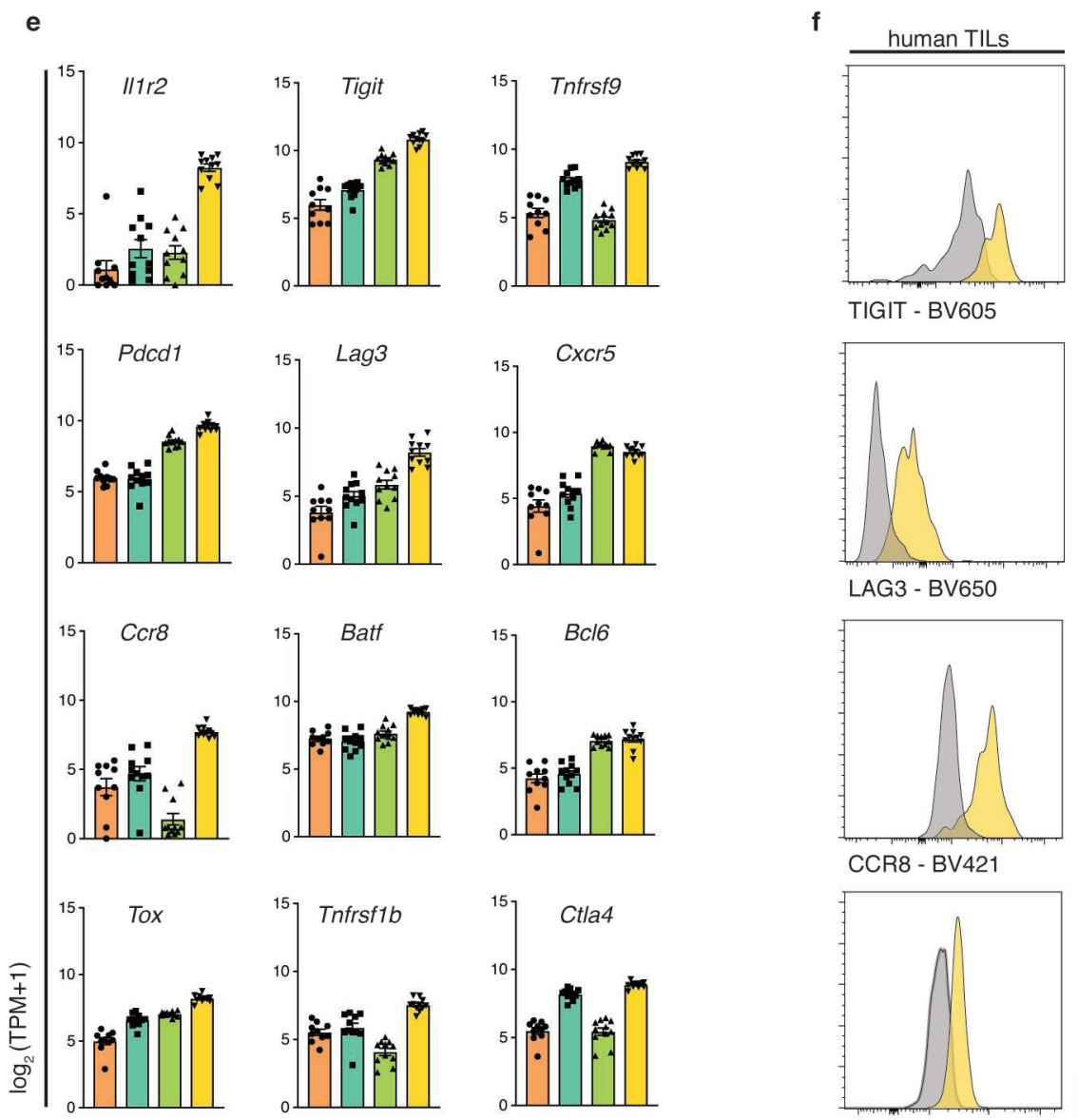

$\mathrm{T}_{\text {EFF }} \square \mathrm{T}_{\text {REG }} \quad \square \mathrm{T}_{\mathrm{FH}} \quad \square \mathrm{T}_{\mathrm{FH}}$

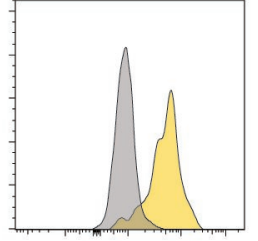

CCR8 - BV421

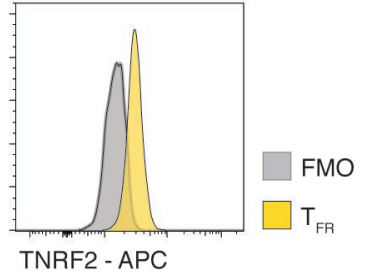


cluster $1\left(T_{R E G}\right)$

- cluster $6\left(\mathrm{~T}_{\mathrm{FR}}\right)$
- Tumor

- LN $\therefore$

๑

$\therefore$

\&

$\%$
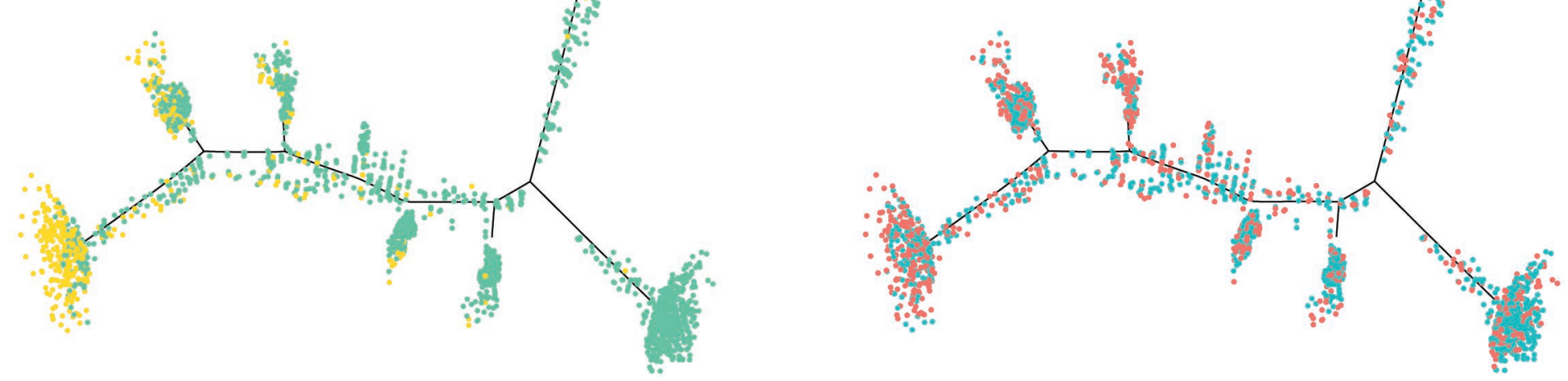

Component 1

b
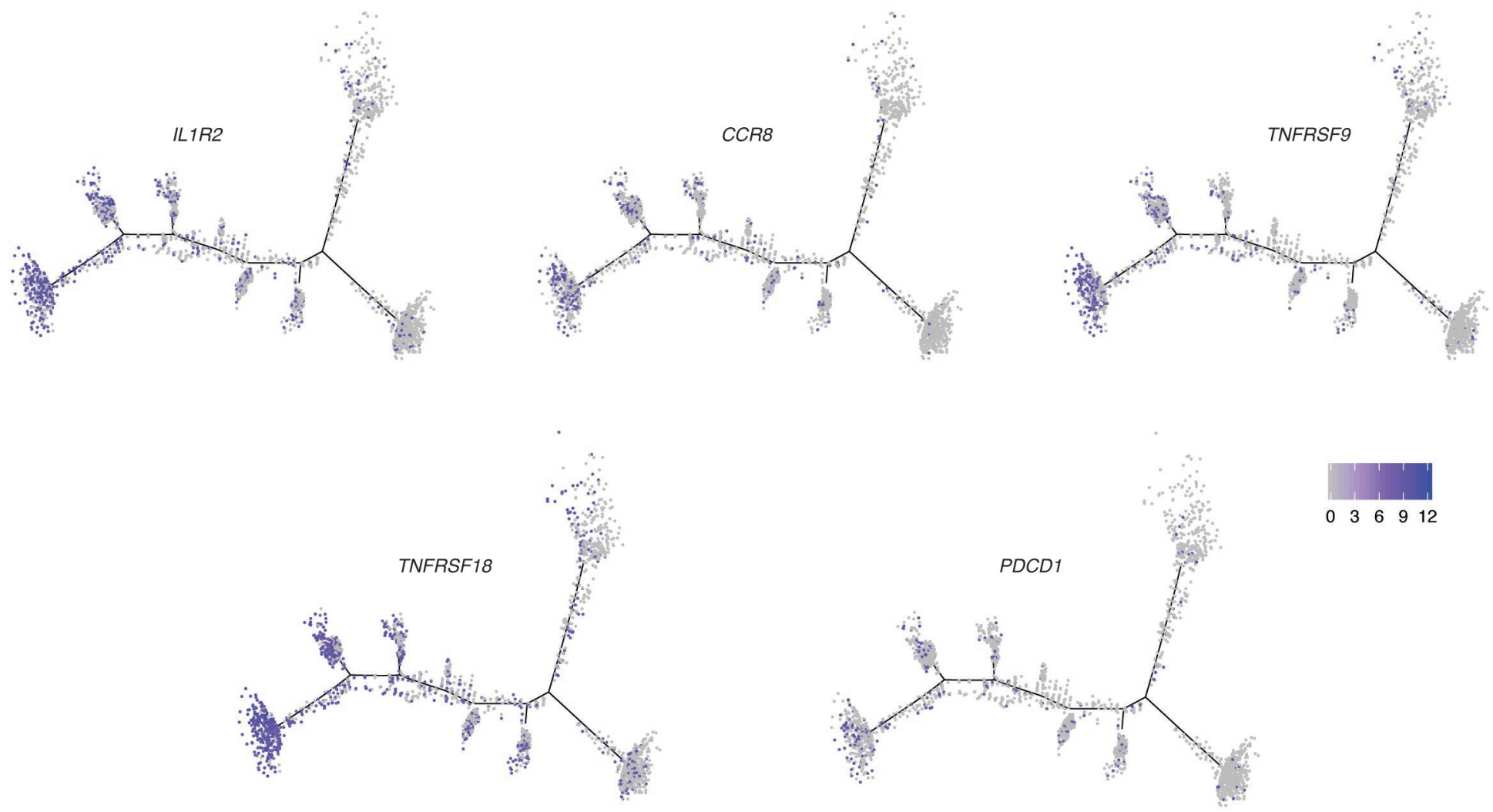

$\begin{array}{llllll}0 & 3 & 6 & 9 & 12\end{array}$ 


\section{a $\quad T_{F R}$ cells}

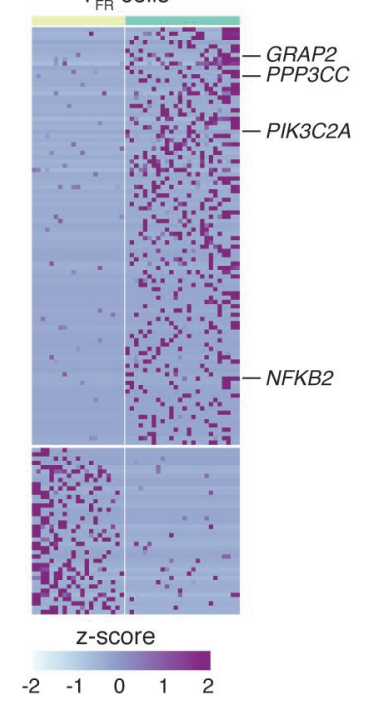

$\begin{array}{lllll}-2 & -1 & 0 & 1 & 2\end{array}$

$\square$ pre anti-PD-1 $\square$ post anti-PD-1

b
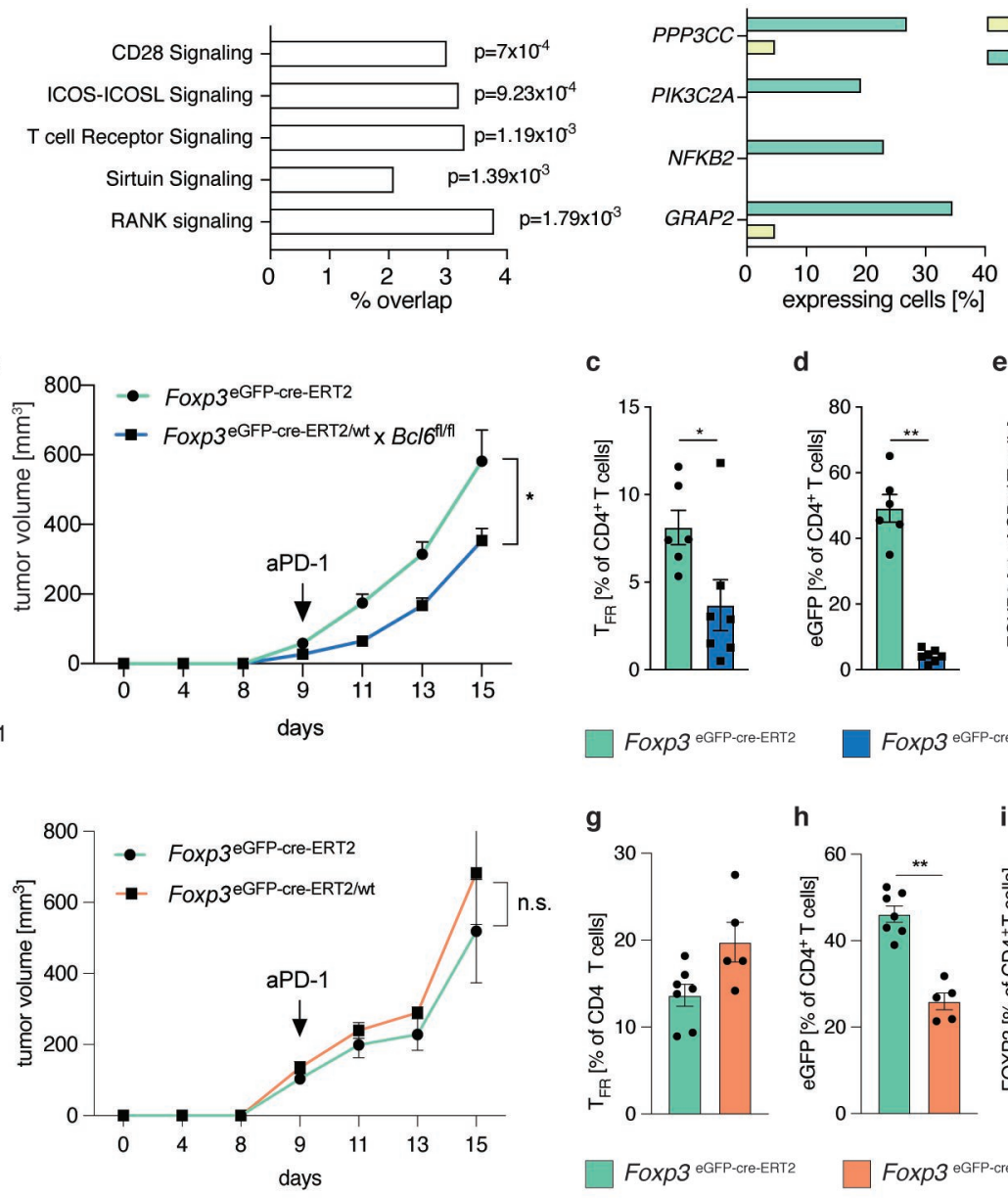

c
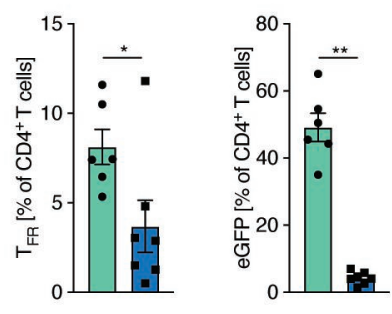

Foxp3 $3^{\text {eGFP-Cre-ERT2 }}$ Foxp3 ${ }^{\text {eGFP-Cre-ERT2/Wt }} \times$ BC/6 $6^{\text {I/III }}$

g

$\square$ pre anti-PD-1

$\square$ post anti-PD-1
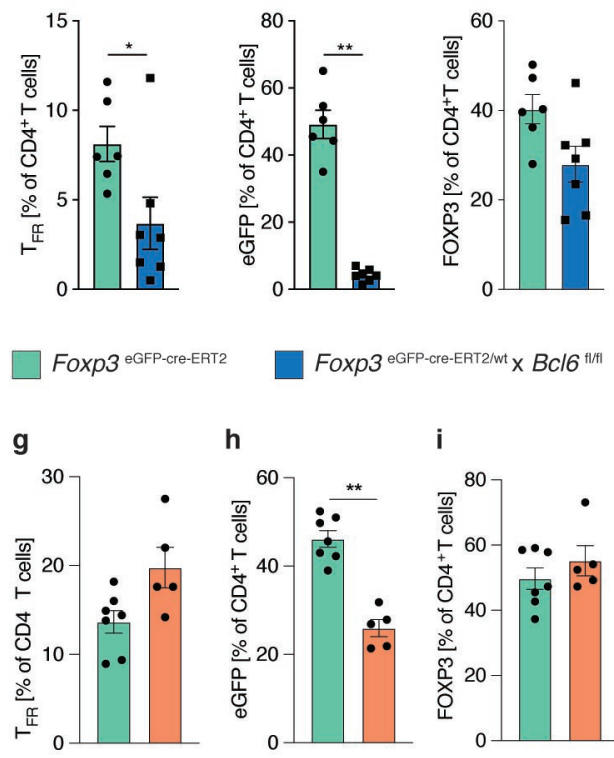

h

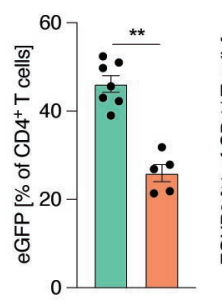

i

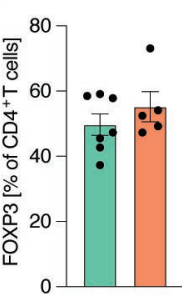

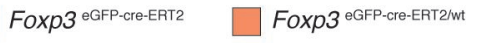


